\title{
One-Sided Difference Approximations for Nonlinear Conservation Laws
}

\author{
By Bjorn Engquist* and Stanley Osher**
}

\begin{abstract}
We analyze one-sided or upwind finite difference approximations to hyperbolic partial differential equations and, in particular, nonlinear conservation laws. Second order schemes are designed for which we prove both nonlinear stability and that the entropy condition is satisfied for limit solutions. We show that no such stable approximation of order higher than two is possible. These one-sided schemes have desirable properties for shock calculations. We show that the proper switch used to change the direction in the upwind differencing across a shock is of great importance. New and simple schemes are developed for which we prove qualitative properties such as sharp monotone shock profiles, existence, uniqueness, and stability of discrete shocks. Numerical examples are given.
\end{abstract}

I. Introduction. Consider a nonlinear hyperbolic conservation law in one space variable

$$
u_{t}+f(u)_{x}=0 \text {. }
$$

A typical solution of (1.1) has discontinuities across curves which separate regions in which the solution is smooth. These discontinuities develop in general even for smooth initial values. It is desirable to obtain a method for the numerical solution of (1.1) which gives a reasonably good approximation for the smooth parts but which also handles discontinuities such as shocks and contact discontinuities correctly. A computed shock should have the right speed, be fairly sharp, and be physically correct, i.e., the entropy condition should be satisfied across it.

Various equivalent characterizations of the entropy condition have been given in the literature; see-e.g. [10]. Here we use the criterion that an admissible solution to (1.1) should satisfy the inequality

$$
\frac{\partial}{\partial t} \frac{u^{2}}{2}+\frac{\partial}{\partial x} F(u)<0
$$

in the sense of distributions where

$$
F(u)=\int_{0}^{u} s f^{\prime}(s) d s .
$$

This inequality is, of course, an equality in regions where the solution $u$ is smooth. For convex $f$ and piecewise continuous $u$, it is well known that $(\mathcal{E})$ is equivalent to the geometric condition that all characteristics flow into a shock.

One-sided or upwind differencing techniques have been used in computational

Received February 6, 1980.

1980 Mathematics Subject Classification. Primary 65M10; Secondary 65M05.

- Partially supported by NSF Grant MCS-79-02735 and the Swedish Natural Science Research Council (NFR-2711-018).

** Partially supported by NSF Grant MCS-78-01252. 
fluid dynamics for many years [19], [13], [17], [18]. Calculations have shown that they are particularly efficient when a shock occurs at a location where the flow changes direction or where supersonic flow becomes subsonic [7]. Errors in onesided approximations at the shock will then not be propagated to the smooth parts of the solution and the computed shock profile stays sharp. The one-sided domain of dependence of upwind differencing which is useful in shock calculations also simplifies the numerical boundary conditions.

For several space dimensions the ADI technique, or dimensional splitting, is generally used [19], [4]. Systems of hyperbolic conservation laws are traditionally approximated by centered difference methods. In recent years upwind differencing has been applied also to these problems [19], [14]. Different components of $f$ are then differenced in opposite directions depending on the sign of the eigenvalues of the Jacobian matrix of $f$.

Although one-sided methods have been extensively used, it was never obvious how to implement the switch in the differencing when the sign of $f^{\prime}$ changed. Nonlinear instabilities and convergence to nonphysical solutions (e.g. expansion shocks) are reported in the literature [7], [9]. It was previously not known how to design one-sided schemes which are guaranteed not to have these defects.

It is the purpose of this paper to present such a method which is of second order away from stagnation points and discontinuities and first order at isolated stagnation points. We will also show that there are no stable one-sided schemes with higher order than two and prove qualitative shock resolution properties for the first order method introduced in [4].

We designed this first order method for a scalar conservation law as a step in approximating the time-dependent small disturbance equation of transonic flow.

$$
2 \Phi_{t x}=\left(K \Phi_{x}-\frac{1}{2}(\gamma+1) \Phi_{x}^{2}\right)_{x}+\Phi_{y y},
$$

where $\Phi$ is the velocity potential, $K$ and $\gamma$ are positive constants. The scheme approximating (1.1) is

$$
u_{j}^{n+1}=u_{j}^{n}-\frac{\Delta t}{\Delta x}\left(\Delta_{+} f_{-}\left(u_{j}^{n}\right)+\Delta_{-} f_{+}\left(u_{j}^{n}\right)\right) .
$$

For convex $f$, we define

$$
f_{+}(u)=f(\max (u, \bar{u})), \quad f_{-}(u)=f(\min (u, \bar{u})),
$$

where $\bar{u}$ is the unique stagnation point, i.e., $f^{\prime}(\bar{u})=0$. For general $f$, we first let

$$
\chi(u) \equiv 1 \quad \text { if } f^{\prime}(u) \geqslant 0, \quad \chi(u) \equiv 0 \quad \text { if } f^{\prime}(u)<0,
$$

and then define

$$
f_{+}(u)=\int_{0}^{u} \chi(s) f^{\prime}(s) d s, \quad f_{-}(u)=\int_{0}^{u}(1-\chi(s)) f^{\prime}(s) d s .
$$

The mesh function $u_{j}^{n}$ approximates $u(x, t)$ at the mesh points $\left(x_{j}, t^{n}\right), x_{j}=j \Delta x$, $t^{n}=n \Delta t$. The operators $\Delta_{+}$and $\Delta_{-}$are respectively forward and backward differences in space $\left(\Delta_{ \pm} u_{j}= \pm\left(u_{j \pm 1}-u_{j}\right)\right)$.

In [4] we proved that this scheme is nonlinearly stable ( $L_{2}$ norm nonincreasing) for a class of functions containing $f(u)=K u-\frac{1}{2}(\gamma+1) u^{2}$ which is the appropriate $f$ for our splitting approximation to (1.2). In fact, the scheme (1.3) is monotone and hence, by the results of [1], [6], the solution $u_{j}^{n}$ must converge in $L_{1}$ 
and the limit solution has no nonphysical shocks. Both signs of $f^{\prime}$ are permitted and, in domains where this sign is constant, (1.3) is the standard first order upwind difference scheme. If, for example, $u_{j}^{n}<\bar{u}$ (for convex $f$ ) for all mesh points involved, we have $\Delta_{-} f_{+}\left(u_{j}^{n}\right) \equiv 0$ and the scheme is

$$
u_{j}^{n+1}=u_{j}^{n}-\frac{\Delta t}{\Delta x} \Delta_{+} f\left(u_{j}^{n}\right)
$$

The difference equation (1.3) is written in conservation form. Lax and Wendroff [11] showed that to satisfy the integral form of the conservation laws it suffices to approximate them by difference equations in conservation form. For example, the well-known one-sided difference approximation to (1.2) of Cole and Murman [12] sometimes gave incorrect shock speeds for limit solutions, until it was put in conservation form [7]. All methods considered in this paper are in conservation form and will thus produce approximations with the right shock speed.

We proved, in [4], existence and uniqueness of the solution approximating a shock for the steady-state difference approximation (1.3). In the present paper we continue this qualitative analysis and prove existence, stability, and monotonicity of sharp discrete shock solutions of (1.3) for the time-dependent approximation.

It is simple to extend the first order one-sided approximation $\Delta_{+} f$ and $\Delta_{-} f$ to second order ones $\left(\Delta_{+}-\frac{1}{2} \Delta_{+}^{2}\right) f$ and $\left(\Delta_{-}+\frac{1}{2} \Delta_{-}^{2}\right) f$. If we let $u_{j}(t)$ approximate $u\left(x_{j}, t\right)$, the first attempt for a second order time-continuous analogue of (1.3) is

$$
\frac{\partial u_{j}}{\partial t}=-\frac{1}{\Delta x}\left(\left(\Delta_{+}-\frac{1}{2} \Delta_{+}^{2}\right) f_{-}\left(u_{j}\right)+\left(\Delta_{-}+\frac{1}{2} \Delta_{-}^{2}\right) f_{+}\left(u_{j}\right)\right) .
$$

It is possible to design a linearly stable scheme based on (1.5) via a LaxWendroff type time-discretization. However, the resulting scheme has severe defects. It produces nonmonotone discrete shock profiles with overshoot in numerical tests. We shall also prove that steady monotone shock solutions do not exist for this scheme.

We propose instead the following scheme for the time-continuous method of lines approximation to (1.1)

$$
\begin{aligned}
\frac{\partial u_{j}}{\partial t}=-\frac{1}{\Delta x}\left(\left(\Delta_{+} f_{-}\left(u_{j}\right)-\frac{1}{2} \Delta_{+}(\right.\right. & \left.\left.f_{-}^{\prime}\left(z_{j}\right) \Delta_{+} u_{j}\right)\right) \\
& \left.+\left(\Delta_{-} f_{+}\left(u_{j}\right)+\frac{1}{2} \Delta_{-}\left(f_{+}^{\prime}\left(w_{j}\right) \Delta_{-} u_{j}\right)\right)\right),
\end{aligned}
$$

where

$$
\begin{array}{r}
z_{j}= \begin{cases}\max \left(u_{j}, u_{j+1}\right) & \text { if } u_{j-1}<\bar{u} \\
\bar{u} & \text { if } u_{j-1}>\bar{u}\end{cases} \\
w_{j}= \begin{cases}\min \left(u_{j}, u_{j-1}\right) & \text { if } u_{j+1}>\bar{u} \\
\bar{u} & \text { if } u_{j+1}<\bar{u}\end{cases}
\end{array}
$$

for $j=2, \ldots, N-2$ with $u_{0}, u_{1}, u_{N-1}$ and $u_{N}$ given.

This scheme is fully one-sided and uses the minimum number of mesh points (three) away from the stagnation point $\bar{u}$. It is of second order accuracy away from $\bar{u}$ and at least of first order near $\bar{u}$. 
We use the energy method for proving the stability of the scheme (1.6). For a class of functions $f$, including $f(u)=K u-\frac{1}{2}(\gamma+1) u^{2}$, we have the strong estimate

$$
\|u(T)\|^{2}<\|u(0)\|^{2} \text {. }
$$

Here || || denotes the usual discrete $L_{2}$ norm either for periodic functions or for the whole real line. An analogous result for more general boundary conditions can be obtained.

For this class of functions $f$, we can also show that any limit solution of (1.6) must satisfy the entropy condition.

Time differencing of Crank-Nicholson type preserves both the unconditional stability and entropy properties and there is strong numerical evidence that a second order explicit Lax-Wendroff time differencing also is stable for CFL number close to the linear stability bound which equals 2 . This time-discretization is given in Section 5 .

We shall also show the somewhat surprising result that the only steady solutions to this second order scheme which agree with a shock as $x \rightarrow \pm \infty$ exactly equal the shock except for at most two points. Moreover, each such profile is monotone with no overshoot. These steady shocks are hence resolved exactly, using our second order scheme (1.6) just as they are for our first order scheme (1.3).

The order of accuracy of the scheme (1.6) is optimal. A one-sided method of lines approximation to the simple equation

$$
u_{t}=a u_{x}
$$

for $a>0$ must have the form

$$
\frac{\partial u_{j}}{\partial t}=\frac{a}{\Delta x} L_{+} u_{j}=\frac{a}{\Delta x} \sum_{k=0}^{p} \alpha_{k} u_{j+k} .
$$

It is of order of accuracy $q$ if, for smooth functions $\psi(x)$,

$$
\frac{1}{\Delta x} L_{+} \psi(x)=\psi_{x}(x)+O\left((\Delta x)^{q}\right)
$$

We will show that an approximation (1.9) cannot be of more than second order of accuracy if it is stable, i.e., if

$$
\|u(T)\|^{2}<C(T)\|u(0)\|^{2} .
$$

The most compact stable second order approximation is given by $L_{+}=\Delta_{+}$ $-\frac{1}{2} \Delta_{+}^{2}$.

The analysis proving this saturation result is given in Section 2. The analogous result for explicit time differencing was proved earlier by Gilbert Strang [15]. The two proofs are based on different principles.

In Section 3 we analyze the second order scheme (1.6). We prove the sharp shock profile, nonlinear stability, and entropy results. A somewhat simpler upwind centered, but not strictly one-sided, scheme is also studied and shown to have all the above mentioned desirable properties for arbitrary convex $f$.

Discrete shocks to the first order accurate approximation (1.3) and to more general monotone schemes are analyzed in Section 4. We also discuss the modified parabolic equation mentioned in [5] and show that discrete shocks for our scheme are generally "closer" to solutions of the hyperbolic equation (1.2) than to solutions 
of this parabolic equation, which contradicts a frequently made conjecture. Our scheme is also shown to contradict estimates on width of transition of discrete shocks in [5]. However, our truncation error leads to a parabolic equation whose viscosity vanishes at sonic points of $f(u)$. The conjectures may be valid for strictly parabolic approximate equations. We also explore briefly the numerical resolution of linear and contact discontinuities.

Finally Section 5 contains results and comments upon some numerical computations.

II. Accuracy in the Smooth Parts of the Solution. When the solution of a nonlinear differential equation is smooth, the convergence of the corresponding difference approximation is, in general, guaranteed by consistency and linear stability [15]. Good stability properties for the linearized equations are necessary but not sufficient for a successful nonlinear method.

We shall analyze the relation between stability and order of accuracy for one-sided approximations to the model problem

$$
\begin{gathered}
u_{t}=a u_{x}, \quad-\infty<x<\infty, t>0, \\
u(x, 0)=\varphi(x),
\end{gathered}
$$

with $a>0$.

We first consider a time-continuous method of lines approximation to (2.1). The solution of $(2.1)$ is approximated by $u_{j}(t),\left(u_{j}(t) \sim u\left(x_{j}, t\right)\right)$. The general form of a one-sided approximation of $(2.1)$ is given by the system of ordinary differential equations

$$
\begin{gathered}
\frac{\partial u_{j}}{\partial t}=\frac{a}{\Delta x} L_{+} u_{j}=\frac{a}{\Delta x} \sum_{k=0}^{p} \alpha_{k} u_{j+k}, \quad t>0, \\
u_{j}(0)=\varphi\left(x_{j}\right), \quad j=0, \pm 1, \pm 2, \ldots .
\end{gathered}
$$

The CFL-condition implies that (2.2) can be stable only if $a>0$. Analogously, the one-sided approximation

$$
L_{-} u_{j}=\sum_{k=0}^{p} \alpha_{k} u_{j-k}
$$

corresponds to negative $a$.

We will assume the method to be consistent with (2.1) which implies

$$
\sum_{k=0}^{p} \alpha_{k}=0, \quad \sum_{k=0}^{p} k \alpha_{k}=1 .
$$

The difference operator $L_{+}$can then be written

$$
L_{+}=\sum_{k=1}^{p} \beta_{k} \Delta_{+}^{k},
$$

with $\beta_{1}=1$. The relation between $\left\{\alpha_{k}\right\}$ and $\left\{\beta_{k}\right\}$ is given by

$$
\begin{gathered}
\alpha_{p}=\beta_{p}, \\
\alpha_{p-1}=\beta_{p-1}-p \beta_{p}, \\
\alpha_{1}=\beta_{1}-2 \beta_{2}+\cdots-(-1)^{p} \beta_{p}, \\
\alpha_{0}=-\beta_{1}+\beta_{2} \cdots+(-1)^{p} \beta_{p} .
\end{gathered}
$$


As in the introduction, we call the approximation (2.2) stable if there exists an estimate

$$
\|u(t)\|^{2}=\Delta x \sum_{j}\left|u_{j}(t)\right|^{2}<C(t)\|u(0)\|^{2}
$$

where $C(t)$ is a function which is bounded independently of $\Delta x$ and $u$. It has the order of accuracy $q$ if, for smooth functions $\psi(x)$,

$$
\frac{1}{\Delta x} L_{+} \psi(x)=\psi_{x}(x)+O\left(\Delta x^{q}\right)
$$

We now present a saturation result which states that there are no stable one-sided methods of arbitrary order of accuracy.

THEOREM 2.1. Stable approximations of type (2.2) can be of at most second order. Moreover, there do exist stable second order approximations and the most compact (smallest $p$ ) is given by $\beta_{1}=1, \beta_{2}=-\frac{1}{2}$ for $p=2$ in (2.4).

Proof. We Fourier transform the differential difference equation (2.2) (2.4) in space. From Parseval's relation, the stability condition (2.6) is equivalent to the inequality

$$
\operatorname{Re}\left[\frac{a}{\Delta x} \sum_{k=1}^{p} \beta_{k}\left(e^{i \theta}-1\right)^{p}\right] \leqslant C, \quad-\pi<\theta<\pi,
$$

for some constant $C$. Since $C$ is independent of $\Delta x$, the stability condition (2.8) can be written

$$
\operatorname{Re}\left[\sum_{k=1}^{p} \beta_{k}(\kappa-1)^{k}\right] \leqslant 0 \text { for }|\kappa|=1 .
$$

Following Dahlquist [3], the unit circle $|\kappa|=1$ is mapped onto the imaginary axis, $\operatorname{Re} z=0$.

$$
z(\kappa)=\frac{\kappa-1}{\kappa+1}, \quad \kappa(z)=-\frac{z+1}{z-1}, \quad \kappa-1=-\frac{2 z}{z-1} .
$$

From (2.9) and (2.10) we get the stability condition

$$
\operatorname{Re}\left[B_{p}(z)\right]<0 \quad \text { for } \operatorname{Re} z=0, \quad B_{p}(z)=\sum_{k=1}^{p} \beta_{k}\left(\frac{2 z}{1-z}\right)^{k}
$$

The function $B_{p}(z)$ is analytic for $\operatorname{Re} z<0$, and for large $|z|$ it is of the form

$$
B_{p}(z)=\bar{\beta}+O\left(|z|^{-1}\right), \quad \bar{\beta}=\sum_{k=1}^{p}(-2)^{k} \beta_{k} .
$$

When (2.11) is valid, the constant $\bar{\beta}$ cannot have a positive real part. The maximum principle for the harmonic function $\operatorname{Re}\left[B_{p}(z)\right]$ implies the equivalent stability condition

$$
\operatorname{Re}\left[B_{p}(z)\right]<0 \text { for } \operatorname{Re} z<0
$$

since $B_{p}(z) \not$ constant. 
Assume now that the order of accuracy is higher than two. We have

$$
\begin{aligned}
\frac{1}{\Delta x} L_{+} \psi(x)= & \beta_{1}\left(\psi_{x}+\frac{\Delta x}{2} \psi_{x x}+\frac{\Delta x^{2}}{6} \psi_{x x x}\right) \\
& +\beta_{2}\left(\Delta x \psi_{x x}+\Delta x^{2} \psi_{x x x}\right)+\beta_{3} \Delta x^{2} \psi_{x x x}+O\left(\Delta x^{4}\right)
\end{aligned}
$$

and hence

$$
\begin{gathered}
\beta_{1}=1, \quad \beta_{2}=-\frac{1}{2}, \quad \beta_{3}=\frac{1}{3} \\
B_{p}(z)=\frac{2 z}{1-z}-\frac{1}{2}\left(\frac{2 z}{1-z}\right)^{2}+\frac{1}{3}\left(\frac{2 z}{1-z}\right)^{3}+O\left(z^{4}\right) \\
=2 z+\frac{2}{3} z^{3}+O\left(z^{4}\right) .
\end{gathered}
$$

Define $\zeta=z^{-1}$ and $G_{p}=B_{p}(z)^{-1}$. The rational function $G_{p}$ then has the analogous properties of (2.13)

$$
\begin{aligned}
& \operatorname{Re}\left[G_{p}(\zeta)\right]<0 \text { for } \operatorname{Re} \zeta<0, \\
G_{p}(\zeta)= & B_{p}\left(\zeta^{-1}\right)^{-1}=\left(2 \zeta^{-1}+\frac{2}{3} \zeta^{-3}+O\left(\zeta^{-4}\right)\right)^{-1} \\
= & \frac{1}{2} \zeta-\frac{1}{6} \zeta^{-1}+O\left(\zeta^{-2}\right)=\frac{1}{2} \zeta+g_{p}(\zeta) .
\end{aligned}
$$

The function $g_{p}$ is analytic for $\operatorname{Re} \zeta<0$ and

$$
\begin{gathered}
\operatorname{Re}\left[g_{p}(\zeta)\right] \rightarrow \operatorname{Re}\left[G_{p}(\zeta)\right] \text { for } \operatorname{Re} \zeta \rightarrow 0 \\
g_{p}(\zeta) \rightarrow 0 \text { for }|\zeta| \rightarrow+\infty
\end{gathered}
$$

Hence we can use (2.15) and the maximum principle for the harmonic function $\operatorname{Re}\left[g_{p}(\zeta)\right]$ to show

$$
\operatorname{Re}\left[g_{p}(\zeta)\right]<0 \text { for } \operatorname{Re} \zeta<0 \text {. }
$$

From (2.16), we have $g_{p}(\zeta)=-\frac{1}{6} \zeta^{-1}+O\left(\zeta^{-2}\right)$ which contradicts (2.17), and the first part of the theorem is proved.

For the second part, we need to show that (2.11) is valid for $\beta_{1}=1, \beta_{2}=-\frac{1}{2}$, $\beta_{k}=0, k>3$.

$$
\begin{aligned}
\operatorname{Re}\left[\frac{2 z}{1-z}-\frac{1}{2}\left(\frac{2 z}{1-z}\right)^{2}\right] & =\operatorname{Re}\left[\frac{2 i \alpha(1-2 i \alpha)}{(1-i \alpha)^{2}}\right] \quad(\text { for } z=i \alpha, \alpha \text { real }) \\
& =-\frac{4 \alpha^{4}}{\left(1+\alpha^{2}\right)^{2}}<0
\end{aligned}
$$

Hence, the scheme is stable and it is easy to see that it is of second order accuracy. There is no second order method with $p=1$ and the second order $p=2$ scheme is unique.

There are several analogies between this theorem and Dahlquist's saturation result for $\boldsymbol{A}$-stable linear multistep approximations to ordinary differential equations [2], [3]. 
Remark. It is easy to construct one-sided difference formulas of any order of accuracy if the constraint of stability is neglected. The difference approximation

$$
\frac{1}{\Delta x} L_{+}=\frac{1}{\Delta x} \sum_{k=1}^{p}\left(-\frac{1}{k}\left(-\Delta_{+}\right)^{k}\right)
$$

is of $p$ th order, but, as we have seen, it is only useful for $p=1$ and $p=2$.

The accuracy of a time-discretization of (2.2) cannot be more than of second order in $\Delta x$ if the scheme is consistent and uniformly stable in $\Delta t$ as $\Delta t \rightarrow 0$. Any solution of (2.2) for fixed $\Delta x$ can be approximated arbitrarily well by the difference scheme, and hence there can be no stability estimate if there is no bound on the solution of (2.2). Compare also the saturation result for explicit methods by Gilbert Strang [15].

For the special and purely theoretical case of a fixed ratio $a \lambda=a \Delta t / \Delta x=1$, the Euler time-discretization with $L_{+}=\Delta_{+}$gives the infinitely accurate method $u_{j}\left(t^{n+1}\right)=u_{j+1}\left(t^{n}\right)$.

The stability analysis for difference approximations of the simple equation (2.1) is far less complex than the corresponding analysis for the approximations of the nonlinear problem (1.1) which we perform in the next section. Since the linear analysis is useful as a necessary condition for more general problems, we will briefly consider some standard time-discretizations of (2.2).

$$
\begin{array}{cl}
u_{j}^{n+1}=\left(1+a \lambda L_{+}\right) u_{j}^{n} & \text { (Euler) } \\
u_{j}^{n+1}=u_{j}^{n-1}+2 a \lambda L_{+} u_{j}^{n} & \text { (leap-frog) } \\
u_{j}^{n+1}=\left(1+a \lambda L_{+}+\frac{(a \lambda)^{2}}{2} \Delta_{+}^{2}\right) u_{j}^{n} & \text { (Lax-Wendroff) } \\
\left(1-\frac{a \lambda}{2} L_{+}\right) u_{j}^{n+1}=\left(1+\frac{a \lambda}{2} L_{+}\right) u_{j}^{n} \quad \text { (Crank-Nicholson) }
\end{array}
$$

We will call the schemes stable if there exist an estimate

$$
\left\|u^{n}\right\| \leqslant C(n \Delta t)\left\|u^{0}\right\|
$$

for the one-step schemes and an estimate

$$
\left\|u^{n}\right\| \leqslant C(n \Delta t)\left(\left\|u_{0}\right\|+\left\|u^{1}\right\|\right)
$$

for (2.20).

THEOREM 2.2. For $L_{+}=\Delta_{+}-\frac{1}{2} \Delta_{+}^{2}$, the schemes (2.19) and (2.20) are unconditionally unstable, (2.21) is conditionally stable for $0<a \lambda<2$, and (2.22) is unconditionally stable.

Proof. The von Neumann stability condition is necessary and sufficient for the scalar one-step methods. It is necessary for the stability of the two-step scheme (2.20). Thus, in order to prove the theorem, we need only to determine the amplification factors $c(\theta)$.

For the scheme (2.19), the amplification factor, i.e., the Fourier transform of the difference operator $\left(1+a \lambda\left(\Delta_{+}-\frac{1}{2} \Delta_{+}^{2}\right)\right)$, is

$$
c(\theta)=1+a \lambda\left(i s(1+2 r)-4 r^{2}\right), \quad|c(\theta)|^{2}=1+(a \lambda \theta)^{2}+O\left(\theta^{4}\right)
$$

where $s=\sin \theta$ and $r=(\sin \theta / 2)^{2}$. 
For (2.20), $c$ is given by

$$
\begin{gathered}
c_{1,2}(\theta)=\alpha \pm \sqrt{1+\alpha^{2}}, \quad \alpha=a \lambda\left(i s(1+2 r)-4 r^{2}\right), \\
\left|c_{2}(\theta)\right|^{2}=1+\frac{a \lambda}{4} \theta^{4}+O\left(\theta^{5}\right) .
\end{gathered}
$$

For (2.21), $c$ is given by

$$
\begin{aligned}
c(\theta) & =1+a \lambda\left(i s(1+2 r)-4 r^{2}\right)+(a \lambda)^{2}\left(4 r^{2}-2 i r s-2 r\right), \\
|c(\theta)|^{2} & =1-4 a \lambda(1-a \lambda)^{2}(2-a \lambda) r^{2}, \quad|c(\theta)|^{2}<1 \text { for } 0<a \lambda<2 .
\end{aligned}
$$

For (2.22), $c$ is given by

$$
c(\theta)=\frac{1+\frac{a \lambda}{2}\left(i s(1+2 r)-4 r^{2}\right)}{1-\frac{a \lambda}{2}\left(i s(1+2 r)-4 r^{2}\right)}, \quad|c(\theta)|<1 .
$$

III. Entropy Production, $L_{2}$ Bound, and Sharp Shock Profiles for the Second Order Scheme. In this section we shall consider the initial value problem for the conservation law

$$
u_{t}+(f(u))_{x}=0, \quad u(x, 0)=u_{0}(x)
$$

Our numerical results will mainly concern $f$ in the class $\mathcal{C}=\left\{f \in C^{3}\left|f^{\prime \prime}(u)\right\rangle\right.$ 0 with a unique $\bar{u}$ such that $f^{\prime}(\bar{u})=0$ \}.

We seek to construct differential-difference approximations to (3.1) having the following properties:

(a) Second order accuracy away from the sonic point $\bar{u}$, at least first order accuracy at $\bar{u}$.

(b) Fully one-sided and using the minimum number of mesh points (three) away from the sonic point.

(c) Nonincreasing $L_{2}$ norm for all solutions.

(d) Limit solutions which must satisfy the entropy condition.

(e) Sharp monotone profiles for zero speed shocks.

As in the introduction, we let $u_{j}(t)$ approximate $u\left(x_{j}, t\right)$.

In order to construct a one-sided scheme, we follow [4] and define

$$
\begin{gathered}
\begin{cases}f_{-}(u)=f(u) & \text { if } u<\bar{u}, \\
f_{-}(u)=f(\bar{u}) & \text { if } u>\bar{u},\end{cases} \\
\begin{cases}f_{+}(u)=f(\bar{u}) & \text { if } u<\bar{u}, \\
f_{+}(u)=f(u) & \text { if } u>\bar{u} .\end{cases}
\end{gathered}
$$

In [3] we constructed a first order accurate scheme having the properties (b)-(e) as follows:

$$
\frac{\partial u_{j}}{\partial t}=-\frac{1}{\Delta x}\left(\Delta_{-} f_{+}\left(u_{j}\right)+\Delta_{+} f_{-}\left(u_{j}\right)\right)
$$

A natural second order one-sided generalization, in view of the linear stability results of the last section, is

$$
\frac{\partial u_{j}}{\partial t}=-\frac{1}{\Delta x}\left[\left(\Delta_{+}-\frac{1}{2} \Delta_{+}^{2}\right) f_{-}\left(u_{j}\right)+\left(\Delta_{-}+\frac{1}{2} \Delta_{-}^{2}\right) f_{+}\left(u_{j}\right)\right]
$$


We have been unable to prove nonlinear stability (or instability) for this scheme. However, in Remark (3.1) below, we shall prove that steady monotone shock solutions of (3.4) do not exist, there must be overshoot. (We also construct all steady solutions to (3.4).)

We propose instead the following scheme:

$$
\begin{aligned}
\frac{\partial u_{j}}{\partial t}=-\frac{1}{\Delta x}\left[\left(\Delta_{+} f_{-}\left(u_{j}\right)\right)-\frac{1}{2} \Delta_{+}\right. & \left(f_{-}^{\prime}\left(z_{j}\right) \Delta_{+} u_{j}\right) \\
& \left.+\left(\Delta_{-} f_{+}\left(u_{j}\right)+\frac{1}{2} \Delta_{-}\left(f_{+}^{\prime}\left(w_{j}\right) \Delta_{-} u_{j}\right)\right)\right],
\end{aligned}
$$

where

$$
\begin{array}{r}
z_{j}= \begin{cases}\max \left(u_{j}, u_{j+1}\right) & \text { if } u_{j-1}<\bar{u}, \\
\bar{u} & \text { if } u_{j-1}>\bar{u}\end{cases} \\
w_{j}= \begin{cases}\min \left(u_{j}, u_{j-1}\right) & \text { if } u_{j+1}>\bar{u} \\
\bar{u} & \text { if } u_{j+1}<\bar{u}\end{cases}
\end{array}
$$

We expand upon the properties (a)-(e) which (3.5) will be shown to have

(a) Smooth solutions to (3.1) satisfy (3.5) with error $O\left((\Delta x)^{2}\right)$ as $\Delta x \rightarrow 0$ at any point $(x, t)$ for which $u(x, t) \neq \bar{u}$. The error is at most $O(\Delta x)$ if $u(x, t)=\bar{u}$ and improves to $O\left((\Delta x)^{2}\right)$ if $f^{\prime \prime}(\bar{u})=0$.

(b) If $u_{j}, u_{j-1} \leqslant \bar{u}$, then the right side of (3.5) involves only $u_{j}, u_{j+1}$, and $u_{j+2}$. Similarly if $u_{j}, u_{j+1} \geqslant \bar{u}$, then only $u_{j}, u_{j-1}$, and $u_{j-2}$ are involved.

(c) Solutions to (3.5) satisfy the a priori estimate for any $t>0$ :

$$
\sum_{j} u_{j}^{2}(t) \Delta x=\|u(t)\|_{2}^{2} \leqslant\|u(0)\|_{2}^{2}
$$

(d) If $u_{j}(t)$ converges boundedly a.e. to $u(x, t)$ as $\Delta x \rightarrow 0$, then $u$ is a weak solution of (3.1) satisfying the entropy inequality

$$
\frac{\partial}{\partial t} \frac{u^{2}}{2}+\frac{\partial}{\partial x} F(u)<0
$$

where

$$
F(u)=\int_{\bar{u}}^{u} s f^{\prime}(s) d s
$$

(e) The only steady solutions of (3.5), satisfying

$$
\lim _{j \rightarrow \infty} u_{j}=u^{R}, \quad \lim _{j \rightarrow-\infty} u_{j}=u^{L},
$$

with $f\left(u^{R}\right)=f\left(u^{L}\right)$ and $f^{\prime}\left(u^{L}\right)>0>f^{\prime}\left(u^{R}\right)$, are of the form: For some $j_{0}$

$$
\begin{array}{ll}
u_{j} \equiv u^{L}, & j<j_{0} \\
u_{j} \equiv u^{R}, & j>j_{0}+1,
\end{array}
$$

and $u_{j_{0}} \in\left(\bar{u}, u^{L}\right], u_{j_{0}+1} \in\left(u^{R}, \bar{u}\right]$, with

$$
f\left(u_{j_{0}}\right)+f\left(u_{j_{0}+1}\right)=f\left(u^{L}\right)+f(\bar{u}) .
$$


The profile thus looks like

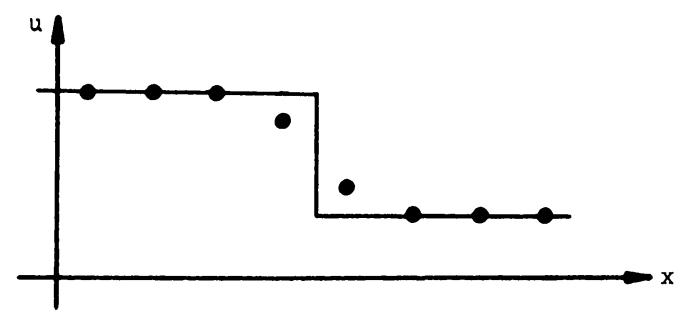

We have

Theorem 3.1. Properties (a), (b), and (e) above are valid for any $f \in \mathcal{C}$ while properties (c) and (d) are valid for $f \in \mathcal{C}$ having the additional property

$$
\begin{aligned}
& \text { (i) } \int_{u}^{v}\left(-1+2\left(\frac{s-v}{u-v}\right)^{2}\right) f^{\prime \prime}(s) d s<0 \text { if } u<v<\bar{u} \\
& \text { (ii) } \int_{u}^{v}\left(-1+2\left(\frac{s-u}{v-u}\right)^{2}\right) f^{\prime \prime}(s) d s<0 \text { if } \bar{u}<u<v .
\end{aligned}
$$

We note that if $f^{\prime \prime}(u)$ is piecewise constant, having a jump only at $u=\bar{u}$, it satisfies (3.7).

We can construct a scheme having properties (a), (c), (d), (e) for arbitrary $f \in \mathcal{C}$, but which is not fully one-sided. However, it is upstream centered (see, e.g., [16], [17]) which helps explain why it also has the steady sharp shock profiles of property (e).

The scheme is

$$
\begin{aligned}
\frac{\partial u_{j}}{\partial t}=-\frac{1}{\Delta x}\left[\left(\Delta_{+} f_{-}\left(u_{j}\right)-\right.\right. & \left.\frac{1}{2} \Delta_{+}\left(f_{-}^{\prime}\left(\zeta_{j}\right) \Delta_{+} u_{j}\right)\right) \\
& \left.+\left(\Delta_{-} f_{+}\left(u_{j}\right)+\frac{1}{2} \Delta_{-}\left(f_{+}^{\prime}\left(\omega_{j}\right) \Delta_{-} u_{j}\right)\right)\right],
\end{aligned}
$$

where this time

$$
\zeta_{j}=\max \left(u_{j-1}, u_{j}, u_{j+1}\right), \quad \omega_{j}=\min \left(u_{j+1}, u_{j}, u_{j-1}\right)
$$

We now have

THEOREM 3.2. Solutions of (3.8) satisfy properties (a), (c), (d), (e) above for arbitrary $f \in \mathcal{C}$. However, property (b) becomes: if $u_{j}, u_{j-1}<\bar{u}$, then the right side of (3.8) involves only $u_{j-1}, u_{j}, u_{j+1}$, and $u_{j+2}$. Similarly, if $u_{j} u_{j+1}>\bar{u}$, then only $u_{j+1}, u_{j}, u_{j-1}$, and $u_{j-2}$ are involved.

Finally we have

Remark (3.1). There exist no monotone steady solutions to (3.4) satisfying $\lim _{j \rightarrow \infty} u_{j}=u^{R}, \lim _{j \rightarrow-\infty} u_{j}=u^{L}$, with $f\left(u^{L}\right)=f\left(u^{R}\right)$ and $f^{\prime}\left(u^{L}\right)>0>f^{\prime}\left(u^{R}\right)$. Any steady solution must have overshoot.

Proof (Theorems (3.1), (3.2) and Remark (3.1)). We begin with the accuracy result, part (a) of both theorems, which is surprisingly complicated to prove. 
Taylor's theorem gives us, for smooth functions $u_{j}(t)=u\left(x_{j}, t\right)$,

(a) $\Delta_{+} f_{-}\left(u_{j}\right)=\Delta x f_{-}^{\prime}\left(u_{j}\right) u_{x}\left(x_{j}\right)$

$$
-\int_{x_{j}}^{x_{j+1}}\left(s-x_{j+1}\right)\left[f_{-}^{\prime \prime}(u(s))\left(u_{x}(s)\right)_{+}^{2}+f_{-}^{\prime}(u(s)) u_{x x}(s)\right] d s
$$

and

(b) $\Delta_{-} f_{+}\left(u_{j}\right)=\Delta x f_{+}^{\prime}\left(u_{j}\right) u_{x}\left(x_{j}\right)$

$$
-\int_{x_{j-1}}^{x_{j}}\left(s-x_{j-1}\right)\left[f_{+}^{\prime \prime}(u(s))\left(u_{x}(s)\right)^{2}+f_{+}^{\prime}(u(s)) u_{x x}(s)\right] d s .
$$

Add and we obtain, for smooth solutions of (3.5)

$$
\begin{aligned}
& \frac{\partial u_{j}}{\partial t}+f^{\prime}\left(u\left(x_{j}\right)\right) u_{x}\left(x_{j}\right) \\
& =\frac{1}{\Delta x}\left[\int_{x_{j}}^{x_{j+1}}\left(s-x_{j+1}\right)\left(f_{-}^{\prime \prime}(u(s))\left(u_{x}(s)\right)+f_{-}^{\prime}(u(s)) u_{x x}(s)\right) d s\right] \\
& \quad+\frac{1}{2} \Delta_{+}\left(f_{-}^{\prime}\left(z_{j}\right) \Delta_{+} u_{j}\right) \\
& +\frac{1}{\Delta x}\left[\int_{x_{j-1}}^{x_{j}}\left(s-x_{j-1}\right)\left(f_{+}^{\prime \prime}(u(s)) u_{x}^{2}(s)+f_{+}^{\prime}(u(s)) u_{x x}(s)\right) d s\right] \\
& \\
& \quad-\frac{1}{2} \Delta_{-}\left(f_{+}^{\prime}\left(w_{j}\right) \Delta_{-} u_{j}\right) .
\end{aligned}
$$

We wish to show that the right side of (3.10) is $O\left((\Delta x)^{2}\right)$ as $\Delta x \rightarrow 0$ if $u\left(x_{j}\right) \neq \bar{u}$ or if $u\left(x_{j}\right)=\bar{u}$ and $f^{\prime \prime}(\bar{u})=0$, and that it is $O(\Delta x)$ if $u\left(x_{j}\right)=\bar{u}$ and $f^{\prime \prime}(\bar{u}) \neq 0$.

If either the first or second case is valid, then $f_{-}, f_{+}$are both $C^{2}$ and piecewise $C^{3}$, so we may integrate by parts once more obtaining

$$
\begin{aligned}
\frac{\partial u_{j}}{\partial t}+ & f^{\prime}\left(u\left(x_{j}\right)\right) u_{x}\left(x_{j}\right) \\
= & -\frac{1}{2} \Delta x\left[f_{-}^{\prime \prime}\left(u\left(x_{j}\right)\right) u_{x}^{2}\left(x_{j}\right)+f_{-}^{\prime}\left(u\left(x_{j}\right)\right) u_{x x}\left(x_{j}\right)-\frac{1}{(\Delta x)^{2}} \Delta_{+}\left(f_{-}^{\prime}\left(z_{j}\right) \Delta_{+} u_{j}\right)\right] \\
& +\frac{1}{2} \Delta x\left[f_{+}^{\prime \prime}\left(u\left(x_{j}\right)\right) u_{x}^{2}\left(x_{j}\right)+f_{+}^{\prime}\left(u\left(x_{j}\right)\right) u_{x x}\left(x_{j}\right)-\frac{1}{(\Delta x)^{2}} \Delta_{-}\left(f_{+}^{\prime}\left(w_{j}\right) \Delta_{-} u_{j}\right)\right] \\
& -\frac{1}{2} \int_{x_{j}}^{x_{j+1}} \frac{\left(s-x_{j+1}\right)^{2}}{\Delta x} \frac{\partial}{\partial s}\left[f_{-}^{\prime \prime}(u(s)) u_{x}^{2}(s)+f_{-}^{\prime}(u(s)) u_{x x}(s)\right] d s \\
& -\frac{1}{2} \int_{x_{j-1}}^{x_{j}} \frac{\left(s-x_{j-1}\right)^{2}}{\Delta x} \frac{\partial}{\partial s}\left[f_{+}^{\prime \prime}(u(s)) u_{x}^{2}(s)+f_{+}^{\prime}(u(s)) u_{x x}(s)\right] d s .
\end{aligned}
$$

The contribution from the two integrals is easily shown to be $O\left((\Delta x)^{2}\right)$. We thus need only show that

$$
f_{-}^{\prime \prime}\left(u\left(x_{j}\right)\right) u_{x}^{2}\left(x_{j}\right)+f_{-}^{\prime}\left(u\left(x_{j}\right)\right) u_{x x}\left(x_{j}\right)-\frac{1}{(\Delta x)^{2}} \Delta_{+}\left(f_{-}^{\prime}\left(z_{j}\right) \Delta_{+} u_{j}\right)=O(\Delta x)
$$

and analogously for $f_{+}$. 
We shall show

(a) $f_{-}^{\prime \prime}\left(u\left(x_{j}\right)\right) u_{x}^{2}\left(x_{j}\right)-\frac{1}{(\Delta x)^{2}}\left(\Delta_{+} f_{-}^{\prime}\left(z_{j}\right)\right) \Delta_{+} u_{j}=O(\Delta x)$

and

(b) $f_{-}^{\prime}\left(u\left(x_{j}\right)\right) u_{x x}\left(x_{j}\right)-\frac{1}{(\Delta x)^{2}} f_{-}^{\prime}\left(z_{j+1}\right) \Delta_{+}^{2} u_{j}=O(\Delta x)$.

Adding these will give us (3.12).

Part (3.13)(b) follows because $\Delta_{+}^{2} u_{j} /(\Delta x)^{2}=u_{x x}\left(x_{j}\right)+O(\Delta x)$ and $f_{-}^{\prime}\left(z_{j+1}\right)=$ $f_{-}^{\prime}\left(u\left(x_{j}\right)\right)+O(\Delta x)$. For part (a), we need

$$
u_{x}\left(x_{j}\right)\left[f_{-}^{\prime \prime}\left(u\left(x_{j}\right)\right) u_{x}\left(x_{j}\right)-\frac{\left(f_{-}^{\prime}\left(z_{j+1}\right)-f_{-}^{\prime}\left(z_{j}\right)\right)}{\Delta x}\right]=O(\Delta x) .
$$

Now if $u$ is monotone on $\left(x_{j}, x_{j+2}\right)$ and $u\left(x_{j}\right) \neq \bar{u}$, then either $z_{j}=u\left(x_{j}\right)$ and $z_{j+1}=u\left(x_{j+1}\right)$ or $z_{j}=u\left(x_{j+1}\right)$ and $z_{j+1}=u\left(x_{j+2}\right)$. In either case, (3.14) is obviously valid. If $u$ is not monotone, then $u_{x}$ vanishes somewhere in this interval and (3.14) is valid with $O\left((\Delta x)^{2}\right)$ as right-hand side. If $u\left(x_{j}\right)=\bar{u}$, then $f^{\prime \prime}(\bar{u})=0$ and the result follows simply.

It remains to show that the scheme is first order accurate at sonic points when $f^{\prime \prime}(\bar{u}) \neq 0$. Then $f_{-}$and $f_{+}$are $C^{1}$ and piecewise $C^{3}$ with a jump in $f^{\prime \prime}$ at $u=\bar{u}$. Equation (3.10) is still valid, and $f_{-}^{\prime}\left(z_{j}\right)=f_{-}^{\prime}\left(z_{j+1}\right)=f_{+}^{\prime}\left(w_{j}\right)=f_{+}^{\prime}\left(w_{j-1}\right)=0$ and the integrands are each easily seen to be $O\left((\Delta x)^{2}\right)$, so the result follows.

For the scheme (3.8), we merely replace $z_{j}$ by $\zeta_{j}$ in (3.10), (3.13), and (3.14), and the result follows with no difficulty.

Next, we obtain the $L_{2}$ estimate, part (c), for both schemes. Multiply both sides of (3.5) by $u_{j} \Delta x$ and sum, arriving at

$$
\begin{aligned}
\frac{\partial}{\partial t}\|u\|_{2}^{2}= & -\sum\left[u_{j} \Delta_{+} f_{-}\left(u_{j}\right)-\frac{1}{2} u_{j} \Delta_{+}\left(f_{-}^{\prime}\left(z_{j}\right) \Delta_{+} u_{j}\right)\right] \\
& -\sum\left[u_{j} \Delta_{-} f_{+}\left(u_{j}\right)+\frac{1}{2} u_{j} \Delta_{-}\left(f_{+}^{\prime}\left(w_{j}\right) \Delta_{-} u_{j}\right)\right]=[\mathrm{I}]+[\mathrm{II}] .
\end{aligned}
$$

Now,

$$
\begin{array}{r}
{[\mathrm{I}]=\sum\left(\int_{u_{j}}^{u_{j+1}}\left(s-u_{j}\right) f_{-}^{\prime}(s) d s+\frac{1}{2} u_{j} \Delta_{+}\left(f_{-}^{\prime}\left(z_{j}\right) \Delta_{+} u_{j}\right)\right)} \\
=\frac{1}{2} \sum\left(\left(\Delta_{+} u_{j}\right)^{2} f_{-}^{\prime}\left(u_{j+1}\right)-\int_{u_{j}}^{u_{j+1}}\left(s-u_{j}\right)^{2} f_{-}^{\prime \prime}(s) d s\right. \\
\left.+\left(\Delta_{-} u_{j}\right)\left(\Delta_{+} u_{j}\right)\left(-f_{-}^{\prime}\left(z_{j}\right)\right)\right),
\end{array}
$$

where we added $\Sigma \int_{u_{j}}^{\mu_{+1}} s f_{-}^{\prime}(s) d s=0$, integrated by parts on the first term, and summed by parts on the second.

Next, we apply Schwarz' inequality to the last term, switch orders of summation, and use the fact that $f_{-}^{\prime}\left(z_{j}\right)<0$ to arrive at

$$
\begin{aligned}
\sum\left(\Delta_{-} u_{j}\right)\left(\Delta_{+} u_{j}\right)\left(-f_{-}^{\prime}\left(z_{j}\right)\right) & \\
& <\frac{1}{2} \sum\left(\Delta_{+} u_{j}\right)^{2}\left(-f_{-}^{\prime}\left(z_{j+1}\right)\right)+\frac{1}{2} \sum\left(\Delta_{+} u_{j}\right)^{2}\left(-f_{-}^{\prime}\left(z_{j}\right)\right) .
\end{aligned}
$$


This tells us

$$
\begin{aligned}
{[\mathrm{I}]<\frac{1}{4} \sum\left(\Delta_{+} u_{j}\right)^{2}\left[2 f_{-}^{\prime}\left(u_{j+1}\right)-f_{-}^{\prime}\left(z_{j+1}\right)-f_{-}^{\prime}\left(z_{j}\right)\right.} \\
\left.-2 \int_{u_{j}}^{u_{j+1}} \frac{\left(s-u_{j}\right)^{2}}{\left(\Delta_{+} u_{j}\right)^{2}} f_{-}^{\prime \prime}(s) d s\right] .
\end{aligned}
$$

If either $u_{j+1}>u_{j}$, or $\bar{u}<u_{j+1}<u_{j}$, the integral appearing above is nonpositive because of convexity, so we need only show the inequality

$$
2 f_{-}^{\prime}\left(u_{j+1}\right)-f_{-}^{\prime}\left(z_{j+1}\right)-f_{-}^{\prime}\left(z_{j}\right)<0 .
$$

Since $z_{j}, z_{j+1} \geqslant u_{j+1},(3.19)$ follows from convexity. Next, we consider the case $u_{j+1}<\bar{u}<u_{j}$. We then must verify

$$
f_{-}^{\prime}\left(u_{j+1}\right)+\int_{u_{j+1}}^{\bar{u}} \frac{\left(s-u_{j}\right)^{2}}{\left(\Delta_{+} u_{j}\right)^{2}} f_{-}^{\prime \prime}(s) d s<0,
$$

or

$$
\left(f_{-}^{\prime}\left(u_{j+1}\right)-f_{-}^{\prime}(\bar{u})\right)+\int_{u_{j+1}}^{\bar{u}} \frac{\left(s-u_{j}\right)^{2}}{\left(\Delta_{+} u_{j}\right)^{2}} f_{-}^{\prime \prime}(s) d s<0
$$

or, finally,

$$
\int_{u_{j+1}}^{\bar{u}}\left[\frac{\left(s-u_{j}\right)^{2}}{\left(\Delta_{+} u_{j}\right)^{2}}-1\right] f_{-}^{\prime \prime}(s) d s<0
$$

which is true by convexity.

The last remaining case is $u_{j+1}<u_{j}<\bar{u}$. Then, if $z_{j+1}>u_{j}$ (which means $u_{j+1} \geqslant u_{j}$ ), we have the estimate

$$
\begin{aligned}
f_{-}^{\prime}\left(u_{j+1}\right)-f_{-}^{\prime}\left(u_{j}\right) & +\int_{u_{j+1}}^{u_{j}} \frac{\left(s-u_{j}\right)^{2}}{\left(\Delta_{+} u_{j}\right)^{2}} f_{-}^{\prime \prime}(s) d s \\
& =2 \int_{u_{j+1}}^{u_{j}}\left(\left(\frac{s-u_{j}}{\Delta_{+} u_{j}}\right)^{2}-1\right) f_{-}^{\prime \prime}(s) d s<0,
\end{aligned}
$$

by convexity. Otherwise, we have $u_{j+1}<u_{j}<0$, and we need to estimate

$$
\begin{aligned}
f_{-}^{\prime}\left(u_{j+1}\right)-f_{-}^{\prime}\left(u_{j}\right) & +2 \int_{u_{j+1}}^{u_{j}} \frac{\left(s-u_{j}\right)^{2}}{\left(\Delta_{+} u_{j}\right)^{2}} f_{-}^{\prime \prime}(s) d s \\
& =2 \int_{u_{j+1}}^{u_{j}}\left[\frac{2\left(s-u_{j}\right)^{2}}{\left(\Delta_{+} u_{j}\right)^{2}}-1\right] f_{-}^{\prime \prime}(s) d s<0,
\end{aligned}
$$

by our special hypothesis.

The proof that $[\mathrm{II}]<0$ follows analogously.

To prove stability for (3.9), we proceed in the same way with $z_{j}$ replaced by $\zeta_{j}$ in (3.18). Since $\zeta_{j}>z_{j}$ and $\zeta_{j+1}>u_{j}$, convexity gives us the desired estimate. 
In order to prove (d), the entropy inequality for limit solutions, we let $\rho(x, t) \in$ $C_{0}^{1}\left(R \times R_{+}\right)$be an arbitrary nonnegative test function. We shall show that solutions to (3.5) satisfy the inequality

$$
\begin{aligned}
&-\sum_{j} \Delta x \int_{0}^{\infty} d t\left[\rho_{t}\left(x_{j}, t\right) \frac{u_{j}^{2}(t)}{2}+\right.\left.\frac{\left(\Delta_{-} \rho\left(x_{j}, t\right)\right)}{\Delta x} F\left(u_{j}(t)\right)\right] \\
&<\sum_{j} \Delta x \int_{0}^{\infty} d t \frac{\left(\Delta_{+} \rho\left(x_{j}, t\right)\right)}{\Delta x}[ u_{j+1} \Delta_{+} f_{+}\left(u_{j}\right)+u_{j} \frac{f_{-}^{\prime}\left(z_{j+1}\right)}{2} \Delta_{+} u_{j+1} \\
&+\frac{u_{j+1} f_{+}^{\prime}\left(w_{j}\right) \Delta_{-} u_{j}}{2}+ \\
&+\frac{1}{4}\left(\Delta_{+} u_{j}\right)^{2} f_{+}^{\prime}\left(w_{j+1}\right) \\
&\left.-\frac{1}{4}\left(\Delta_{+} u_{j}\right)^{2} f_{-}^{\prime}\left(z_{j+1}\right)\right] .
\end{aligned}
$$

Then, as $\Delta x \rightarrow 0$ if $u\left(x_{j}, t\right) \rightarrow u(x, t)$ boundedly a.e., the above right side $\rightarrow 0$ and the left side $\rightarrow-\iint\left(\rho_{t}\left(u^{2} / 2\right)+\rho_{x} F(u)\right) d x d t$ by the Lebesgue dominated convergence theorem. Since $\rho \geqslant 0$ is arbitrary, inequality (3.6) is immediate.

To obtain (3.25) we multiply (3.5) by $\rho\left(x_{j}, t\right) u_{j} \Delta x$, sum and integrate, then add

$$
\begin{aligned}
-\sum \Delta x \int_{0}^{\infty} d t \frac{\left(\Delta_{-} \rho\left(x_{j}, t\right)\right)}{\Delta x} F\left(u_{j}(t)\right) \\
\quad=\sum_{j} \Delta x \int_{0}^{\infty} d t \frac{\rho\left(x_{j}, t\right)}{\Delta x} \int_{u_{j}}^{u_{j+1}} s f^{\prime}(s) d s
\end{aligned}
$$

to both sides, arriving at (suppressing the $t$ dependence)

Left side of (3.25)

$$
\begin{aligned}
= & \sum \rho\left(x_{j}\right)\left[\int_{u_{j}}^{u_{j+1}}\left(s-u_{j}\right) f_{-}^{\prime}(s) d s+\frac{1}{2} u_{j} \Delta_{+}\left(f_{-}^{\prime}\left(z_{j}\right) \Delta_{+} u_{j}\right)\right] \\
& +\sum \rho\left(x_{j}\right)\left[\int_{u_{j}}^{u_{j+1}}\left(s-u_{j+1}\right) f_{+}^{\prime}(s) d s-\frac{1}{2} u_{j} \Delta_{-}\left(f_{+}^{\prime}\left(w_{j}\right) \Delta_{-} u_{j}\right)\right] \\
& +\sum\left(\Delta_{+} \rho\left(x_{j}\right)\right) u_{j+1} \Delta_{+} f_{+}\left(u_{j}\right) \\
= & {[\mathrm{I}]_{\rho}+[\mathrm{II}]_{\rho}+[\mathrm{III}]_{\rho}^{\prime} . }
\end{aligned}
$$

We shall call all $o(1)$ terms [III $]_{\rho}^{\nu}$, numbering them as they arise. Integrate and sum by parts in $[\mathrm{I}]_{\rho}$ arriving at

$$
\begin{aligned}
{[\mathrm{I}]_{\rho}=} & \sum \frac{\rho\left(x_{j}\right)}{2}\left[\left(\Delta_{+} u_{j}\right)^{2} f_{-}^{\prime}\left(u_{j+1}\right)-\int_{u_{j}}^{u_{j+1}}\left(s-u_{j}\right)^{2} f_{-}^{\prime \prime}(s) d s\right. \\
& \left.+\left(\Delta_{-} u_{j}\right)\left(\Delta_{+} u_{j}\right)\left(-f_{-}^{\prime}\left(z_{j}\right)\right)\right] \\
& +\sum \frac{\left(\Delta_{-} \rho\left(x_{j}\right)\right)}{2} u_{j-1} f_{-}^{\prime}\left(z_{j}\right) \Delta_{+} u_{j} .
\end{aligned}
$$


Call the last term above [III $]_{\rho}^{2}$. Schwarz' inequality gives us

$$
\begin{aligned}
\sum \frac{\rho\left(x_{j}\right)}{2}\left(\Delta_{-} u_{j}\right)\left(\Delta_{+} u_{j}\right)\left(-f_{-}^{\prime}\left(z_{j}\right)\right) & \\
< & \sum \frac{\left(\Delta_{+} u_{j}\right)^{2}}{4}\left[\rho\left(x_{j}\right)\left(-f_{-}^{\prime}\left(z_{j}\right)-f_{-}^{\prime}\left(z_{j+1}\right)\right)\right] \\
& -\sum \frac{\left(\Delta_{+} u_{j}\right)^{2}}{4} f_{-}^{\prime}\left(z_{j+1}\right) \Delta_{+} \rho\left(x_{j}\right) \\
& =\sum \frac{\left(\Delta_{+} u_{j}\right)^{2}}{4} \rho\left(x_{j}\right)\left(-f_{-}^{\prime}\left(z_{j}\right)-f_{-}^{\prime}\left(z_{j+1}\right)\right)+[\text { III }]_{\rho}^{3} .
\end{aligned}
$$

Thus we have

$$
\begin{array}{r}
{[\mathrm{I}]_{\rho}<\sum \frac{\rho\left(x_{j}\right)}{4}\left(\Delta_{+} u_{j}\right)^{2}\left[2 f_{-}^{\prime}\left(u_{j+1}\right)-f_{-}^{\prime}\left(z_{j}\right)-f_{-}^{\prime}\left(z_{j+1}\right)\right.} \\
\left.\quad-2 \int_{u_{j}}^{u_{j+1}}\left(\frac{s-u_{j}}{\Delta_{+} u_{j}}\right)^{2} f_{+}^{\prime \prime}(s) d s\right]+[\mathrm{III}]_{\rho}^{2}+[\mathrm{III}]_{\rho}^{3} .
\end{array}
$$

It is also easy to show

$$
\begin{aligned}
{[\mathrm{II}]_{\rho}<\sum \frac{\rho\left(x_{j}\right)}{4}\left[-2 f_{+}^{\prime}\left(u_{j}\right)+f_{+}^{\prime}\left(w_{j}\right)+f_{+}^{\prime}\left(w_{j+1}\right)\right.} \\
\left.\quad-\int_{u_{j}}^{u_{j+1}} \frac{\left(s-u_{j+1}\right)^{2}}{\Delta_{+} u_{j}} f_{+}^{\prime \prime}(s) d s\right]+[\mathrm{III}]_{\rho}^{4}+[\mathrm{III}]_{\rho}^{s},
\end{aligned}
$$

where

$$
\begin{aligned}
& {[\mathrm{III}]_{\rho}^{4}=\sum \frac{\Delta_{+} \rho\left(x_{j}\right)}{2} u_{j+1} f_{+}^{\prime}\left(w_{j}\right) \Delta_{-} u_{j}} \\
& {[\mathrm{III}]_{\rho}^{5}=\sum \frac{\Delta_{+} \rho\left(x_{j}\right)}{4}\left(\Delta_{+} u_{j}\right)^{2} f_{+}^{\prime}\left(w_{j+1}\right) .}
\end{aligned}
$$

The first term in brackets in both (3.29) and (3.30) has already been shown to be nonpositive. Thus, (3.25) follows from (3.27), (3.29), (3.30), and (3.31).

The proof of part (d) for solutions of (3.8) follows in the same fashion.

Next, we prove part (e) of both theorems and the related Remark (3.1).

Summing (3.5) from $-\infty$ to $j$ shows us that steady solutions satisfy

$$
f_{-}\left(u_{j+1}\right)-\frac{1}{2} f_{-}^{\prime}\left(z_{j+1}\right) \Delta_{+} u_{j+1}+f_{+}\left(u_{j}\right)+\frac{1}{2} f_{+}^{\prime}\left(w_{j}\right) \Delta_{-} u_{j}=f(\bar{u})+f\left(u^{L}\right) .
$$

Let $j_{0}$ be such that $u_{j}>\bar{u}$ for $j<j_{0}, u_{j_{0}+1}<\bar{u}$. Then, for $j<j_{0}-1$, (3.32) becomes

$$
f\left(u_{j}\right)+\frac{1}{2} f^{\prime}\left(w_{j}\right) \Delta_{-}\left(u_{j}\right)=f\left(u^{L}\right)
$$

or

$$
\Delta_{-} u_{j}=\frac{2\left(f\left(u^{L}\right)-f\left(u_{j}\right)\right)}{f^{\prime}\left(w_{j}\right)}
$$


Thus, if for some $j_{1}<j_{0}-1$ we have $u_{j_{1}}>u^{L}$, then $\Delta_{-} u_{j_{1}}<0$ or $u^{L}<u_{j_{1}}<u_{j_{1}-1}$. Repeating this gives us $u^{L}<u_{j_{1}}<u_{\nu}$ if $\nu<j_{1}$, which is impossible. Similarly, $u_{j_{1}}<u^{L}$ is also impossible; thus $u_{j} \equiv u^{L}$ for $j<j_{0}-1$.

Next, we claim $u_{j_{0}+2}<\bar{u}$, for suppose this is false. Then (3.32), for $j=j_{0}+1$, gives us

$$
f_{-}(\bar{u})+f_{+}(\bar{u})=f_{-}(\bar{u})+f\left(u^{L}\right)
$$

or

$$
f_{+}(\bar{u})=f_{+}\left(u^{L}\right)
$$

which is a contradiction.

We also claim $u_{j}<\bar{u}$ if $j>j_{0}+2$. Suppose there is $j_{1}>j_{0}+2$ for which $u_{j_{1}}>\bar{u}$ while $u_{j}<\bar{u}$ for $j_{0}+2 \leqslant j \leqslant j_{1}$. Let $j=j_{1}-1$ in (3.32). Then we have (3.34) which is a contradiction. It now follows that $u_{j} \equiv u^{R}$ for $j>j_{0}+2$ in the same way that it was shown that $u_{j} \equiv u^{L}$ for $j<j_{0}-1$, and (3.32) is valid for all $j$ except $j=j_{0}$. For this value we have

$$
f_{-}\left(u_{j_{0}+1}\right)+f_{+}\left(u_{j_{0}}\right)=f(\bar{u})+f\left(u^{L}\right),
$$

and for each $u_{j_{0}} \in\left(\bar{u}, u^{L}\right]$, there exists one $u_{j_{0}+1} \in\left(u^{R}, \bar{u}\right]$ solving this. Thus part (e) of Theorem (3.1) is proven. The same proof works for Theorem (3.2).

To prove Remark (3.1), we examine steady solutions of (3.4) which must satisfy

$$
f_{-}\left(u_{j+1}\right)-\frac{1}{2} \Delta_{+} f_{-}\left(u_{j+1}\right)+f_{+}\left(u_{j}\right)+\frac{1}{2} \Delta_{-} f_{+}\left(u_{j}\right)=f(\bar{u})+f\left(u^{L}\right) .
$$

Define $j_{0}$ as above. Then, for $j<j_{0}-2$ (notice, not $j_{0}-1$ ), we have

$$
f_{+}\left(u_{j}\right)+\frac{1}{2} \Delta_{-} f_{+}\left(u_{j}\right)=f\left(u^{L}\right)
$$

or

$$
\Delta_{-} u_{j}=\frac{2\left(f\left(u^{L}\right)-f\left(u_{j}\right)\right)}{\left(\Delta_{-} f\left(u^{j}\right)\right) / \Delta_{-} u_{j}} .
$$

So, as above, $u_{j} \equiv u^{L}$ for $j<j_{0}-2$. We again claim $u_{j_{0}+2}<\bar{u}$, because if not, we may take $j=j_{0}+1$ in (3.36) giving us

$$
-\frac{1}{2}\left(f\left(u_{j_{0}+3}\right)-f(\bar{u})\right)-\frac{1}{2}\left(f_{+}\left(u_{j_{0}}\right)-f(\bar{u})\right)=f\left(u^{L}\right)-f(\bar{u}),
$$

but the left side is nonpositive and the right side is positive, another contradiction. We suppose there exists $j_{1}>j_{0}+1$ for which $u_{j_{1}}>\bar{u}$, while $u_{j}<\bar{u}$ for $j+2<j<$ $j_{1}$. Letting $j=j_{1}-1$ in (3.36) gives us (3.38) with $j_{0}$ replaced by $j_{1}-2$, giving us the same contradiction. Thus, we have

$$
\begin{aligned}
& u_{j} \equiv u^{L}, \quad j<j_{0}-2, \\
& u_{j} \equiv u^{R}, \quad j>j_{0}+3,
\end{aligned}
$$

and it remains to solve (3.36) for $j=j_{0}-1, j_{0}$, and $j_{0}+1$.

We may normalize so that $\bar{u}=0=f(\bar{u})$. These equations become
(a) $\frac{3}{2} f_{+}\left(u_{j_{0}-1}\right)-\frac{1}{2} f_{-}\left(u_{j_{0}+1}\right)=\frac{3}{2} f\left(u^{L}\right)$
(b) $-\frac{1}{2} f_{+}\left(u_{j_{0}-1}\right)+\frac{3}{2} f_{+}\left(u_{j_{0}}\right)+\frac{3}{2} f_{-}\left(u_{j_{0}+1}\right)-\frac{1}{2} f_{-}\left(u_{j_{0}+2}\right)=f\left(u^{L}\right)$,
(c) $-\frac{1}{2} f_{+}\left(U_{j_{0}}\right)+\frac{3}{2} f_{-}\left(u_{j_{0}+2}\right)=\frac{3}{2} f\left(u^{L}\right)$. 
In order that a discrete shock exist, we need a solution to this with $u_{j_{0}-1}, u_{j_{0}}>\bar{u}$; $u_{j_{0}+1}, u_{j_{0}+2}<\bar{u}$. Solving this system of equations in terms of $f_{+}\left(u_{j_{0}-1}\right)$ and $f\left(u^{L}\right)$ gives us

$$
\begin{aligned}
f_{+}\left(u_{j_{0}}\right) & =\frac{9}{2} f\left(u^{L}\right)-3 f_{+}\left(u_{j_{0}-1}\right), \\
f_{-}\left(u_{j_{0}+1}\right) & =-3 f\left(u^{L}\right)+3 f_{+}\left(u_{j_{0}-1}\right), \\
f_{-}\left(u_{j_{0}+2}\right) & =\frac{5}{2} f\left(u^{L}\right)-f_{+}\left(u_{j_{0}-1}\right) .
\end{aligned}
$$

The following inequalities are easily shown to be necessary and sufficient for existence of a solution:

$$
\begin{array}{ll}
\frac{3}{2} f\left(u^{L}\right)>f_{+}\left(u_{j_{0}-1}\right) \geqslant f\left(u^{L}\right), & \frac{3}{2} f\left(u^{L}\right)>f_{+}\left(u_{j_{0}}\right)>0, \\
\frac{3}{2} f\left(u^{L}\right)>f_{-}\left(u_{j_{0}+1}\right) \geqslant 0, & \frac{3}{2} f\left(u^{L}\right)>f_{-}\left(u_{j_{0}+2}\right)>f\left(u^{L}\right) .
\end{array}
$$

Thus there must be overshoot. Prescribing $u_{j_{0}-1}$ in the interval $\left[u^{L}, f_{+}^{-1}\left(\frac{3}{2} f\left(u^{L}\right)\right)\right]$ gives us a unique solution.

IV. Discrete Shocks for the First Order Scheme. In this section we shall analyze discrete shocks and contact discontinuities for the first order accurate monotone difference approximation to (3.1) of the form

$$
\begin{aligned}
u_{j}^{n+1} & =u_{j}^{n}-\lambda\left[\Delta_{+} f_{-}\left(u_{j}^{n}\right)+\Delta_{-} f_{+}\left(u_{j}^{n}\right)\right]=G\left(u_{j+1}^{n}, u_{j}^{n}, u_{j-1}^{n}\right) \\
& =u_{j}^{n}-\lambda\left(h\left(u_{j+1}^{n}, u_{j}^{n}\right)-h\left(u_{j}^{n}, u_{j-1}^{n}\right)\right),
\end{aligned}
$$

where

$$
f_{+}(u)=\int_{0}^{u} \chi(s) f^{\prime}(s) d s, \quad f_{-}(u)=\int_{0}^{u}(1-\chi(s)) f^{\prime}(s) d s,
$$

for

$$
\begin{array}{ll}
\chi(u)=1 & \text { if } f^{\prime}(u)>0, \\
\chi(u)=0 & \text { if } f^{\prime}(u)<0,
\end{array}
$$

with the CFL condition $\lambda\left|f^{\prime}(u)\right|<1$.

We shall prove existence, stability, monotonicity, and an ordering principle for discrete shock solutions of (4.1) following [8]. Jennings' results in [8] apply only to strictly monotone schemes, i.e., if

$$
u_{j}^{n+1}=G\left(u_{j+k}^{n}, \ldots, u_{j-l}^{n}\right),
$$

then $G_{\mu}>0$ for $\mu=-l, \ldots, k$. Our scheme is only weakly monotone, i.e., $G_{u}>0$; thus we need to use some of its special properties in order to prove these results.

Another difficulty is the existence of numerous errors in Jennings' work. In particular, his stability proof has to be completely redone. A new proof was recently obtained by James Ralston; we shall give it below (Jennings' existence proof was also cleaned up by Ralston with some minor modifications; we omit the details).

Our difference scheme will be shown to approximate steady shocks with infinite resolution and also to approximate a wide class of moving shocks with infinite resolution in front of the shock. What is perhaps more surprising is that contact and linear discontinuities can also, in some cases, be resolved exactly, thus contradicting much numerical evidence, e.g. [5]. 
Finally, we analyze the truncation error equation for (4.1). It has been conjectured in several places, e.g. [5], that solutions to difference schemes are closer to solutions of the truncation error equation than to solutions of the hyperbolic conservation law (3.1). We shall show that this is sometimes false for (4.1) using the discrete shocks discussed above. We also show that previously conjectured estimates [5] for the spreading of discrete shocks are not valid in this case.

A discrete shock solution of (4.1) moving with speed $s$ satisfies the difference approximation

$$
u_{j-s \lambda}=G\left(u_{j+1}, u_{j}, u_{j-1}\right) .
$$

The minimal domain on which (4.3) makes sense consists of functions defined on the linear span over the integers of $\eta=s \lambda$ and 1. Call the closure of this set $\mathscr{L}_{\eta}$. If $\eta$ is rational, $\mathcal{L}_{\eta}$ is discrete, if $\eta$ is irrational, then $\mathcal{L}_{\eta}$ is the entire real line. $L_{1}\left(\mathcal{C}_{\eta}\right)$ is the space of absolutely integrable functions on $\mathscr{L}_{\eta}$ with the usual measure. Let the solution of (4.3) satisfy

$$
\text { (a) } \lim _{j \rightarrow-\infty} u_{j}=u^{L}, \quad \lim _{j \rightarrow \infty} u_{j}=u_{j}^{R},
$$

the Rankine-Hugoniot relation

$$
\text { (b) } s\left(u^{R}-u^{L}\right)=f\left(u^{R}\right)-f\left(u^{L}\right) \text {, }
$$

and Oleinik's condition E

$$
\text { (c) } \frac{f(u)-f\left(u^{R}\right)}{u-u^{R}}<\frac{f\left(u^{L}\right)-f\left(u^{R}\right)}{u^{L}-u^{R}}
$$

for $u$ strictly between $u^{R}$ and $u^{L}$.

Our first result concerns existence and ordering of discrete shocks. For convenience only, we shall take $u^{R}<u^{L}$ in what follows.

THEOREM 4.1. If $|\eta|<1$, then for each $u_{0} \in\left(u^{R}, u^{L}\right)$ there is a function continuous on $\mathcal{L}_{\eta}$ taking on the value $u_{0}$ at $j=0$ which satisfies (4.3), (4.4) and which is a monotone nonincreasing function of $j$. These shocks obey the following ordering principle: if $s \neq 0$, or if $s=0$ for convex $f \in \mathcal{C}$, then, if $u_{0}>v_{0}$ for two such discrete shocks, it follows that $u_{j} \geqslant v_{j}$ for all $j$.

We next have a stability result for discrete shocks.

Theorem 4.2. Suppose $\eta \neq 0$ is rational, or $\eta=0$ and $f \in \mathcal{C}$, and the initial function $\left\{u_{j}^{0}\right\}$ has the properties

(a) $\Sigma_{j>0}\left|u_{j}^{0}-u^{R}\right|<\infty$,

(b) $\Sigma_{j<0}\left|u_{j}^{0}-u^{L}\right|<\infty$,

(c) $u_{j} \in\left[u^{L}, u^{R}\right]$ for all $j$.

Then the sequence

$$
u_{j}^{n+1}=G\left(u_{j+1}^{n}, u_{j}^{n}, u_{j-1}^{n}\right), \quad n=0,1, \ldots,
$$

converges as $n \rightarrow \infty$ to the discrete shock $v_{j}$ satisfying $\Sigma\left(v_{j}-u_{j}^{g}\right)=0$.

We also have sharp shock profiles without overshoot as follows. 
Theorem 4.3. (a) Suppose the shock speed $s>0$ and $f^{\prime}\left(u^{R}\right)<0$. Then the monotone discrete shock solution to (4.3), (4.4) has the property that there exists $j_{0}$ with $u_{j} \equiv u^{R}$ for $j>j_{0}$. In fact, if $u_{0}$ is such that $f^{\prime}(u)<0$ for all $u \in\left[u^{R}, u_{0}\right]$, then $j_{0}<1-s \lambda$.

(b) If $s<0$ and $f^{\prime}\left(u^{L}\right)$, then there exists $j_{0}$ with $u_{j} \equiv u^{L}$ for $j<j_{0}$, and if $f^{\prime}(u)>0$ for all $u \in\left[u_{0}^{R}, u^{L}\right]$, then $j_{0} \geqslant-1+s \lambda$.

(c) If $s=0$ for convex $f \in \mathcal{C}$, then there exists $j_{0}$ with $u_{j} \equiv u^{L}$ for $j<j_{0}, u_{j} \equiv u^{R}$ for $j>j_{0}+1$, and $u_{j_{0}}, u_{j_{0}+1}$ solve $f_{-}\left(u_{j_{0}+1}\right)+f_{+}\left(u_{j_{0}}\right)=f_{-}\left(u^{L}\right)+f_{+}\left(u^{L}\right)$ with $f_{-}^{\prime}\left(u_{j_{0}}\right)$ $=0=f_{+}^{\prime}\left(u_{j_{0}+1}\right)$.

In order to prove the stability result, we shall need the following result of Ralston.

THEOREM 4.4 (RALSTON). Suppose $\eta$ is rational and the initial function $\left\{u_{j}^{0}\right\}$ has the properties of Theorem 4.2. Then, for a strictly monotone scheme, the sequence

$$
u_{j}^{n+1}=G\left(u_{j+k}^{n}, \ldots, u_{j-k}^{n}\right)
$$

converges as $n \rightarrow \infty$ to the discrete shock $v_{j}$ satisfying $\Sigma\left(v_{j}-u_{j}^{9}\right)=0$.

Before proving these theorems we present the following remarks.

Remark (4.1). The existence part of Theorem 4.1 can be generalized to any weakly monotone scheme

$$
u_{j}^{n+1}=G\left(u_{j+k}^{n}, \ldots, u_{j-l}^{n}\right)
$$

having the property that for any $\varepsilon>0$ sufficiently small there exists a $G^{e}\left(u_{j+k}, \ldots, u_{j-l}\right)$ such that the resulting scheme is strictly monotone and $G^{e}\left(u_{j+k}, \ldots, u_{j-l}\right) \rightarrow G\left(u_{j+k}, \ldots, u_{j-l}\right)$ as $\varepsilon \searrow 0$. For example, the well-known Lax-Friedrichs scheme

$$
w_{j}^{n+1}=\frac{1}{2}\left(w_{j+1}^{n}+w_{j-1}^{n}\right)-\frac{\lambda}{2}\left(\Delta_{0} f\left(w_{j}^{n}\right)\right)=G\left(w_{j+1}^{n}, w_{j}^{n}, w_{j-1}^{n}\right)
$$

is only weakly monotone because $G_{0}=0$, yet, if we require the strict CFL condition $\lambda\left|f^{\prime}(w)\right|<1$, we may take $G^{e}-G=-\lambda_{\varepsilon} \Delta_{+} \Delta_{-} w_{j}$ for $\varepsilon$ sufficiently small, to prove existence of discrete shocks for rational $\eta$. (Notice that we added negative dissipation in this rather peculiar case in order to construct the strictly monotone $G^{e}$.) It was pointed out by Ralston that, regarded as an algorithm of the form

$$
u_{j}^{n+2}=G\left(w_{j+2}^{n}, w_{j}^{n}, w_{j-2}^{n}\right),
$$

the Lax-Friedrichs scheme is strictly monotone. It is easy to use this along with Jennings' existence and uniqueness results to show that uniqueness, monotonicity, and the ordering principle are in general false for the Lax-Friedrichs scheme for $\eta$ rational.

Remark (4.2). If a discontinuity obeys Oleinik's condition E,

$$
\frac{f(u)-f\left(u^{R}\right)}{u-u^{R}}<\frac{f\left(u^{L}\right)-f\left(u^{R}\right)}{u^{L}-u^{R}} \text { for } u \in\left(\min \left(u^{R}, u^{L}\right), \max \left(u^{R}, u^{L}\right)\right),
$$

it is usually called a shock. If the left side identically equals the right, it is usually called a linear discontinuity. Finally if equality holds somewhere in the interval, it is usually called a contact discontinuity. It is widely stated, e.g. [5], that good numerical resolution of contact discontinuities is difficult to obtain, that there is 
more spreading than for shocks. This is true in general. However, our first order scheme (4.1) may have numerical steady solutions approximating linear and contact discontinuities with infinite resolution. If we take, for example, a function which looks like

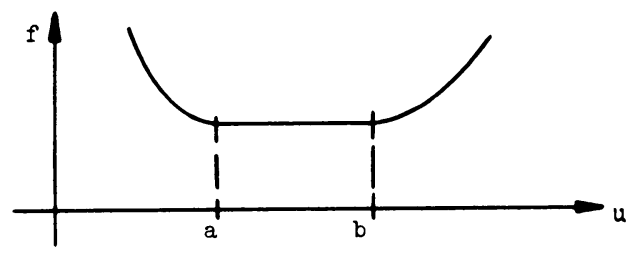

with $f$ constant for $a<u<b, f^{\prime}(u)>0$ if $u>b, f^{\prime}(u)<0$ if $u<a$, then a discrete steady solution is

$$
u_{j} \equiv b \quad \text { for } j<j_{0}, \quad u_{j} \equiv a \quad \text { for } j>j_{0} .
$$

We next address ourselves to an analysis of the truncation error equation. For a difference scheme approximating (3.1) of the usual form (4.2) with $C^{2}$ and piecewise $C^{3}$ coefficients, a truncation error analysis shows that all smooth solutions of (4.1) satisfy

$$
\text { (a) } \begin{array}{r}
u\left(x_{j}, t+\Delta t\right)-G\left(u\left(x_{j+k}, t\right), \ldots, u\left(x_{j-l}, t\right)\right) \\
=(\Delta t)^{2}\left[\mathscr{B}(u, \lambda) u_{x}\right]_{x}+O\left((\Delta t)^{3}\right), \text { where }
\end{array}
$$

(b) $\mathscr{B}(u, \lambda)=\frac{1}{2 \lambda^{2}} \sum_{j=-l}^{k} j^{2} G_{j}(u, u, u, \ldots, u)-\frac{1}{2}\left(f^{\prime}(u)\right)^{2}$.

It has been pointed out [5] that solutions of monotone schemes such as (4.1) of ten behave very much like solutions of the modified parabolic equation

$$
u_{t}+(f(u))_{x}=\Delta t\left[\Re(u, \lambda) u_{x}\right]_{x}
$$

where $\mathscr{B}(u, \lambda)$ is defined in $(4.5)(\mathrm{b})$. In fact, it is conjectured that discrete shocks are frequently closer to solutions of (4.6) than to solutions of (3.1). It was also conjectured [5] that the width of transition of these discrete shocks can be approximated by

$$
w\left(u_{-}, u_{+}\right) \approx \lambda \int_{u_{-}}^{u_{+}} \frac{\mathscr{B}(w, \lambda) d w}{f(w)-f\left(u^{L}\right)-s\left(w-u^{L}\right)},
$$

where $w\left(u_{-}, u_{+}\right)$measures the number of cells occupied by values between $u_{-}$and $u_{+}$. We have

Remark (4.3). Both of the above conjectures are sometimes false for the scheme (4.1).

We prove this as follows. First in order that the functions in (4.1) be $C^{2}$ and piecewise $C^{3}$, we take $f \in \mathcal{C}$ with $f^{\prime \prime}(\bar{u})=0$. (We could make the coefficients in (4.1) $C^{k}$ for arbitrary $k$ by requiring that a sufficiently high number of derivatives of $f$ vanish at $u=\bar{u}$.) For convenience, we again take $\bar{u}=f(\bar{u})=0$. For our scheme

$$
\mathscr{B}(u, \lambda)=\frac{1}{2 \lambda}\left|f^{\prime}(u)\right|\left[1-\lambda\left|f^{\prime}(u)\right|\right]>0,
$$


and (4.6) becomes

$$
u_{t}+(f(u))_{x}=\frac{\Delta x}{2}\left(\left|f^{\prime}(u)\right|\left(1-\lambda\left|f^{\prime}(u)\right|\right) u_{x}\right)_{x}
$$

We look for a viscous profile, a solution of (4.9) of the form $u((x-s t) / \Delta x)=$ $u(r)$ satisfying $u(\infty)=u^{R}, u(-\infty)=u^{L}$ and $s=\left(f\left(u^{R}\right)-f\left(u^{L}\right)\right) /\left(u^{R}-u^{L}\right)$ satisfies the strict entropy condition E. Such a solution solves the ordinary differential equation

$$
-s\left(u-u^{L}\right)+f(u)-f\left(u^{L}\right)=\frac{1}{2}\left|f^{\prime}(u)\right|\left(1-\lambda\left|f^{\prime}(u)\right|\right) u^{\prime} .
$$

Take a shock for which $s \geqslant 0$ and $u^{R}<0<u^{L}$. We impose the condition $u(0)=0$ to fix it, and solve (4.10) obtaining an implicit expression for the solution

$$
\begin{array}{ll}
r=\frac{1}{2} \int_{0}^{u} d w \frac{\left(f^{\prime}(w)+\lambda\left(f^{\prime}(w)\right)^{2}\right)}{f(w)-f\left(u^{R}\right)-s\left(w-u^{R}\right)}, & r>0, \\
r=\frac{1}{2} \int_{u}^{0} d w \frac{f^{\prime}(w)-\lambda\left(f^{\prime}(w)\right)^{2}}{f(w)-f\left(u^{L}\right)-s\left(w-u^{L}\right)}, & r<0 .
\end{array}
$$

In Theorem 4.3 we have shown for $s>0$ that $v_{j} \equiv u^{R}$ for $j>1-s \lambda$ for the discrete shock satisfying these conditions at $\pm \infty$. We interpolate if necessary and let

$$
u_{j}^{n}=v(j \Delta x, n \Delta t)=v(\Delta x(j-s \lambda n))=v_{j-s \lambda n}=v_{r} \equiv u^{R}
$$

for $r>1-s \lambda$, which is very different from the function $u(r)$ defined implicitly in (4.11). Thus, for $(x-s t) / \Delta x \geqslant 1-s \lambda$, the exact solution to the difference equation coincides with the solution to the hyperbolic differential equation and is far from any solution to the parabolic truncation error equation. Suppose, in Harten's expression (4.7), we take $0>u_{-}>u_{+}>u^{R}$. We have shown $0<w\left(u_{-}, u_{+}\right)<1-$ $s \lambda<1$, while the expression he has is

$$
\frac{1}{2} \int_{u_{-}}^{u_{+}} \frac{\left|f^{\prime}(w)\right|\left[1-\lambda\left|f^{\prime}(w)\right|\right] d w}{f(w)-s w-\left(f\left(u^{R}\right)-s u^{R}\right)}
$$

Clearly, as $u_{+} \searrow u^{R}$, this expression approaches $+\infty$ while the true number is always less than one.

We also note that, as $s \searrow 0$, these discrete shocks can easily be shown to converge to the discrete shock for $s=0$ which gives infinite resolution on both sides. Thus, for $s$ close to zero, these discrete shocks give good resolution even behind the shock. See the next section for numerical verification of this.

The remainder of this section will be devoted to proving Theorems 4.1-4.4.

Proof (Theorem 4.1). We modify Eq. (4.1) to make the right side strictly monotone.

$$
u_{j-s \lambda}=G^{e}\left(u_{j+1}^{e}, u_{j}^{e}, u_{j-1}^{e}\right)=G\left(u_{j+1}^{e}, u_{j}^{e}, u_{j-1}^{e}\right)+\varepsilon \lambda \Delta_{+} \Delta_{-} u_{j}^{e}
$$

for $\varepsilon>0$ sufficiently small. An explicit calculation gives us

$$
\begin{aligned}
G_{1}^{e} & =-\lambda f_{-}^{\prime}\left(u_{j+1}\right)+\varepsilon \lambda>\varepsilon \lambda>0, \\
G_{0}^{e} & =1+\lambda\left[f_{-}^{\prime}\left(u_{j}\right)-f_{+}^{\prime}\left(u_{j}\right)-2 \varepsilon\right] \\
& =1-\lambda\left[\left|f^{\prime}\left(u_{j}\right)\right|+2 \varepsilon\right]>1-\mu_{0}-2 \lambda \varepsilon>0, \\
G_{-1}^{e} & =\lambda f_{+}^{\prime}\left(u_{j-1}\right)+\varepsilon \lambda>\varepsilon \lambda>0,
\end{aligned}
$$


if

$$
0<\varepsilon<\frac{1-\mu_{0}}{2 \lambda} \text { for } \mu_{0}=\sup \lambda\left|f^{\prime}(u)\right|<1 .
$$

We first construct traveling waves for $\eta=p / q, p, q$ mutually prime. Iterating (4.5) $q$ times gives us

$$
\begin{aligned}
& \text { (a) } u_{j-p}^{e}=G^{e, q}\left(u_{j+q}^{e}, \ldots, u_{j-q}^{e}\right), \\
& \text { (b) } u_{j}^{e}=G^{e, q}\left(u_{j+p+q}^{e}, \ldots, u_{j+p-q}^{e}\right) .
\end{aligned}
$$

$G^{e, q}$ is monotone increasing in its arguments.

By Theorem (1) of [8], for any $u_{0} \in\left(u^{R}, u^{L}\right)$, there exists a unique $\left\{u_{j}^{e}\right\}_{-\infty}^{\infty}$ which takes on the value $u_{0}$ at $j=0$ and satisfies (4.4), (4.15)(b) and is monotone decreasing. Now we let $\varepsilon \searrow 0$ for $j=1 . u_{0}>u_{1}^{e}>u^{R}$, hence a subsequence $u_{1}^{e_{1}} \rightarrow u_{1}^{0}$. Repeating this procedure gives us a sequence $u_{j}^{e^{(0)}}$ such that $u_{j}^{e^{(0)}} \rightarrow u_{j}$ for each $j$. It is clear that $u_{j}$ is monotone nonincreasing in $j$ and satisfies (4.14)(b) for $\varepsilon=0$. It remains only to show that $u_{\infty}=u^{L}, u_{-\infty}=u^{R}$. Since $u_{j}$ is monotone, it follows that $u_{\infty}$ exists and $u^{L}>u_{0}>u_{\infty}>u^{R}$. For any $\varepsilon^{(v)}$ and $j_{0}$ we have

$$
\sum_{j=j_{0}}^{\infty}\left(u_{j}^{e^{(v)}}-u_{j+p}^{e^{(v)}}\right)=-\lambda \sum_{j=j_{0}}^{\infty}\left(h_{j+p+1}^{e^{(v)}, q}-h_{j+p}^{e^{(v)}, q}\right),
$$

using the conservation form of $G$ and hence $G^{e, q}$. Using consistency we have

$$
u_{j_{0}}^{e^{(v)}}+\cdots+u_{j_{0}+p-1}^{e^{(v)}}-p u^{R}=\lambda\left(h_{j_{0}+p}^{e^{(v)}}-q f\left(u^{R}\right)\right) .
$$

Now let $\varepsilon^{(v)} \searrow 0$. We have, for any $\delta>0$, by taking $j_{0}$ sufficiently large

$$
\left|\left(u_{\infty}-u^{R}\right)-s\left(f\left(u_{\infty}\right)-f\left(u^{R}\right)\right)\right|<\delta .
$$

Hence,

$$
s=\frac{f\left(u_{\infty}\right)-f\left(u^{R}\right)}{u_{\infty}-u^{R}} \quad \text { for } u_{\infty} \in\left[u^{R}, u_{0}\right],
$$

and hence $u_{\infty}=u^{R}$ by condition E. The proof that $u_{-\infty}=u^{L}$ follows similarly.

Next we prove the ordering principle. The key idea is an observation of Jennings [8],-if $u$ and $v$ are two discrete shock solutions with the same limits at $\pm \infty$, then for all $j$

$$
\left|u_{j}-v_{j}\right|=\sum_{\nu=-q}^{q} G_{\nu}^{q}\left|u_{j+\nu+p}-v_{j+\nu+p}\right|,
$$

where each partial derivative $G_{\nu}^{q}$ is evaluated at a point on the straight line connecting the pair of $(2 q+1)$ vectors $\left(u_{j+p+q}, \ldots, u_{j+p-q}\right)$ and $\left(v_{j+p+q}, \ldots, v_{j+p-q}\right)$ such that

$$
u_{j}-v_{j}=\sum_{\nu=-q}^{q} G_{\nu}^{q}\left(u_{j+\nu+p}-v_{j+\nu+p}\right) .
$$

First we suppose $p>0$. Condition E implies $f^{\prime}(u)>0$ for $u^{L}>u \geqslant u^{L}-\delta$ for some $\delta>0$. Our scheme is such that $G_{0}^{q}>0$. Thus (4.19), (4.20) imply that if $u_{0}>v_{0}$, then $u_{-k p}>v_{-k p}$ for all positive integers $k$. Choose $k_{1}$ so large that $u_{j}, v_{j}>u^{L}-\delta$ if $j<-k_{1} p$. Suppose there exists $j_{1}<-k_{1} p$ with $u_{j_{1}}<v_{j_{1}}$. Our prior analysis shows there is $j_{2}$ such that $j_{1}-p<j_{2}<j_{1}$ with $u_{j_{2}}>v_{j_{2}}$. Consider (4.19), 
(4.20) for $j=j_{1}-p$. Our scheme is such that $G_{\nu}^{q}>0$ here for $\nu=$ $-q, \ldots, 0$, hence $u_{j_{1}}-v_{j_{1}}$ and $u_{j_{2}}-v_{j_{2}}$ cannot be of opposite signs. This contradiction proves $u_{j} \geqslant v_{j}$ for all $j<-k_{1} p$. Now if $u_{j_{3}}<v_{j_{3}}$ for some $j_{3}$, it follows that $u_{j_{3}-k p}<v_{j_{3}-k p}$ for all positive integers $k$, contradicting the above result. Hence $u_{0}>v_{0} \Rightarrow u_{j} \geqslant u_{j}$.

An analogous argument works for $p<0$.

If $p=0$, we need the additional hypothesis of convexity, $f \in \mathcal{C}$. The ordering principle and, in fact, a uniqueness result both follow from part (c) of Theorem 4.3 proven below.

In order to construct discrete shocks for irrational $\eta>0$, we choose a sequence of rationals $s_{i} \lambda=\eta_{i} \rightarrow \eta$. Fix $u_{i}^{R}=u^{R}$ and let $u_{i}^{L}$ vary so that the sharp entropy condition remains valid. This can be done by taking $u_{i}^{L}>u^{L}$ and redefining $f$ if necessary for $u_{i}^{L}>u>u^{L}$. The functions $u_{j}^{i}$ for certain $j$ rational can be extended to piecewise constant monotone nondecreasing functions $u(x)$ continuous on the left. We can pass to a subsequence if necessary so $u^{i}(x) \rightarrow w(x)$ on a dense subset of the line. The limit is monotone, hence it can be extended to a function defined on the whole real line and continuous on the left. Also $w_{0}=u_{0}$. The fact that $w_{\infty}=u^{R}, w_{-\infty}=u^{L}$ can be proven using condition $\mathrm{E}$ as in the proof of the first part of this theorem.

It is clear that Eq. (4.3) is valid for all $j$ in $R^{1}$. Hence existence is proven.

Suppose $w(x)$ and $z(x)$ are two such solutions continuous from the left. Then it is easy to see that Jennings' observation (4.19), (4.20) is valid even in the irrational case (for $q=1$ here). If $w(0)>z(0)$, then there exists some $\delta>0$ for which $w(x)>z(x)$ for each interval $-\delta-k s \lambda<x<-k s \lambda$, for all positive integers. Let $x_{0}$ be such that $f_{-}^{\prime}(w(x)) \equiv f_{-}^{\prime}(z(x))$ if $x \leqslant x_{0}$. It also follows that if $x_{1}<x_{0}$, with $w\left(x_{1}\right)>z(x)$, then $w\left(x_{1}-\nu\right) \geqslant z\left(x_{1}-\nu\right)$ and $w\left(x_{1}-\nu s \lambda\right)>z\left(x_{1}-\nu s \lambda\right)$, for all positive integers $\nu$. We use the fact that $G_{\mu}^{q}>0$ in (4.19), (4.20) if $j<x_{1}-\nu s \lambda$ and $q<\mu<0$. Hence, $w(x) \geqslant z(x)$ for any $x=x_{1}=-k_{1} s \lambda-k_{2}$ for $k_{1}, k_{2}$ positive integers and all $x_{1}$ in an interval $-\delta-k_{3} s \lambda<x_{1}<k_{3} s \lambda$ for $k_{3}$ a fixed sufficiently large positive integer. It is easy to show that this means there exists some positive integer $k_{4}$ for which $w(x) \geqslant z(x)$ for all $x<-k_{4}$. By a now familiar argument, it follows that $w(x) \geqslant z(x)$ for all $x$ and the ordering principle is valid.

If $w(x)$ and $z(x)$ are two discrete shocks continuous from the left, then the integral

$$
h(t)=\int_{-\infty}^{\infty}(z(x+t)-w(x)) d x
$$

is continuous. By the ordering principle, there exists some $t_{0}$ for which $h\left(t_{0}\right)=0$, and, furthermore, $z\left(x+t_{0}\right) \equiv w(x)$. Since $u_{0}$ was arbitrary in $\left(u^{R}, u^{L}\right)$, it follows that $w(x)$ takes on every value in this interval and is hence continuous.

Proof (Theorem (4.4) (Ralston)). The proof begins by first assuming the initial vector $\left\{u_{j}^{0}\right\}$ lies between two discrete shocks $\left\{\underline{v}_{j}\right\}$ and $\left\{\bar{v}_{j}\right\}$. For monotone difference operators $T$,

it follows that

$$
u_{j}^{n+1}=\left\{T u^{n}\right\}_{j-\eta}=\left\{T^{n+1} u^{0}\right\}_{j-\eta(n+1)}
$$

$$
\underline{v}_{j}<\left(T^{k} u\right)_{j}<\bar{v}_{j}
$$


We can find a vector $\left\{w_{j}\right\}$ and a subsequence $T^{k^{\prime} u}$ such that

$$
\left\|T^{k_{l} u}-w\right\|_{1}=\sum_{j}\left|\left(T^{k^{\prime} u}\right)_{j}-w_{j}\right| \rightarrow 0 \quad \text { as } k_{l} \rightarrow \infty
$$

(For this we need Jennings' result that any discrete shock $\left\{\tilde{v}_{j}\right\}$ approaches $u^{L}$ and $u^{R}$ exponentially in $j$ as $j \rightarrow \pm \infty$. This result is easily extended to our weakly monotone scheme.)

Since $T$ is an $L_{1}$ contraction, we have for any discrete shock

(a) $\left\|T^{k_{1}+\mu_{u}}-T^{\mu_{v}}\right\|_{1} \leqslant\left\|T^{k_{1}} u-\tilde{v}\right\|_{1} \rightarrow\|w-\tilde{v}\|_{1}$

$$
\begin{aligned}
& \qquad \begin{array}{l}
\downarrow \\
\left\|T_{w}^{\mu_{w}}-\tilde{v}\right\|_{1}
\end{array} \\
& \text { and } \\
& \left\|T^{k_{l+1}-k_{l}-1} T^{k_{l}+\mu_{u}}-T^{k_{l+1}-k_{t}-1} \tilde{v}\right\|_{1}<\left\|T^{k_{l}+\mu_{u}}-\tilde{v}\right\|_{1} \rightarrow\left\|T^{\mu_{w}}-\tilde{v}\right\| \\
& \qquad \\
& \qquad\|w-\tilde{v}\|_{1} .
\end{aligned}
$$

So for any discrete shock $\tilde{v}$ and any positive integer $\mu$

$$
\left\|T^{\mu_{w}}-\tilde{v}\right\|_{1}=\|w-\tilde{v}\|_{1}
$$

For the particular discrete shock $\left\{v_{j}\right\}$ having the property that $\Sigma\left(v_{j}-u_{j}^{0}\right)=0$, it follows that $\Sigma\left(v_{j}-T^{n} u_{j}^{9}\right)=\Sigma\left(v_{j}-u_{j}^{9}\right)=0$ for any $n$, hence $\Sigma\left(v_{j}-w_{j}\right)=0$. Thus, if $w_{j} \neq v_{j}$, it follows that $\left(w_{j}-v_{j}\right)$ is not always of the same sign.

Now

$$
\begin{aligned}
\left|\left(T^{\mu} w\right)_{j}-\left(T^{\mu}\right)_{j}\right|= & \left|G^{\mu}\left(w_{j+k+\eta}, \ldots, w_{j-k+\eta}\right)-G^{\mu}\left(v_{j+k+\eta}, \ldots, v_{j-k+\eta}\right)\right| \\
= & \left|\sum_{\nu=-k}^{k} G_{\nu}^{\mu}\left(w_{\nu+j+\eta}-v_{\nu+j+\eta}\right)\right|<\sum_{\nu=-k}^{k} G_{\nu}^{\mu}\left|w_{\nu+j+\eta}-v_{\nu+j+\eta}\right| \\
= & \left|w_{j+k+\eta}-v_{j+k+\eta}\right|-\lambda \nabla h_{j+\eta}^{\mu} \cdot\left|\vec{w}_{j+\eta}-\vec{v}_{j+\eta}\right| \\
& +\lambda \nabla h_{j+\eta-1}^{\mu} \cdot\left|\vec{w}_{j+\eta-1}-\vec{v}_{j+\eta-1}\right|,
\end{aligned}
$$

where $\vec{w}_{j}=\left\{w_{j+k}, \ldots, w_{j-k+1}\right\}$.

Summing gives us

$$
\left\|T^{\mu_{w}}-u\right\|_{1} \leqslant\|w-u\|_{1}
$$

but by (4.22) this is an equality. Hence, equality holds in (4.23). If $w_{j_{0}}-u_{j_{0}}$ and $w_{j_{1}}-u_{j_{1}}$ are of opposite signs, we may choose $j$ appropriately and $\mu$ so large that $w_{j_{0}}-u_{j_{0}}$ and $w_{j_{1}}-u_{j_{1}}$ appear on the right in (4.23). By strict monotonicity (the only place we use it), strict inequality must hold in (4.23) which is a contradiction. Thus, we have proven the theorem for this class of initial data.

Given a vector $\left\{u_{j}^{0}\right\}$ satisfying the hypotheses of this theorem, we shall show below that, for any $\varepsilon>0$, there exists $u^{e}$ bounded above and below by discrete shocks with $\left\|u^{\varepsilon}-u^{0}\right\|<\varepsilon / 2$, and $\Sigma\left(u_{j}^{e}-u_{j}^{g}\right)=0$.

Then, for any such $u^{\varepsilon}$, we have

$$
\left\|T^{n} u^{\varepsilon}-v\right\|_{1}<\varepsilon / 2 \text { if } n>N(\varepsilon),
$$

and, since $T$ is an $L_{1}$ contraction, it follows that

$$
\left\|T^{n} u^{0}-v\right\|_{1}<\varepsilon \quad \text { if } n>N(\varepsilon) .
$$


It remains only to construct $u^{e}$. We first choose $M$ such that

$$
\sum_{|j|>M}\left|u_{j}^{0}-v_{j}\right|<\varepsilon, \quad v_{M}<u^{R}+\varepsilon, \quad v_{-M}>u^{L}-\varepsilon .
$$

Next, by translating $v_{j}$, we choose discrete shocks $\bar{v}^{\varepsilon}, \underline{v}^{e}$ with

$$
\sum_{|j|<M}\left(u^{L}-\bar{v}_{j}^{e}\right)<\varepsilon, \quad \sum_{|j|<M}\left(\underline{v}_{j}^{e}-u^{R}\right)<\varepsilon .
$$

We then define $u^{\varepsilon}$ as

$$
u_{j}^{e}=v_{j}, \quad|j|>M, \quad u_{j}^{e}=\max \left(\underline{v}_{j}^{e}, \min \left(u_{j}, \bar{v}_{j}^{e}\right)\right), \quad|j|<M .
$$

We claim that $\left|\Sigma_{|j| \neq M}\left(v_{j}-u_{j}^{e}\right)\right|<3 \varepsilon$. This follows from (4.25), (4.27) and the fact that $\Sigma\left(v_{j}-u_{j}^{g}\right)=0$. Thus, if $\varepsilon<\left(u^{L}-u^{R}\right) / 6$, we can find $u_{-M}^{e}, u_{M}^{e}$ in the intervals $\left[u^{L}-3 \varepsilon, v_{-M}\right]$ and $\left[v_{M}, u^{R}+3 \varepsilon\right]$, respectively, so that $\Sigma\left(v_{j}-u_{j}^{e}\right)=0$.

Next, we note that

$$
\left\|u-u^{\varepsilon}\right\|_{1}<\varepsilon+2 \varepsilon+6 \varepsilon=9 \varepsilon .
$$

Finally, we claim that for any $j, \bar{v}_{j}^{\varepsilon} \geqslant u_{j}^{\varepsilon} \geqslant \underline{v}_{j}^{e}$.

For $|j|<M$, this is immediate. For $|j| \geqslant \vec{M}$, it is a consequence of the fact that we can take $\bar{v}_{j}^{e}>v_{j} \geqslant \underline{v}_{j}^{\varepsilon}$ for any $j$ which follows from the ordering principle for discrete shocks.

Proof (Theorem (4.2)). Clearly we need only prove this result for $\left\{u_{j}^{0}\right\}$ between two discrete shocks $\left\{\underline{v}_{j}\right\}$ and $\left\{\bar{v}_{j}\right\}$. Define $w_{j}=\lim \left(T^{k_{n}} u_{j}\right)$ as before, and again let $u_{j}$ be the traveling wave solution satisfying $\Sigma\left(v_{j}-u_{j}^{0}\right)=0$. Equation (4.22) is still valid for this weakly monotone scheme, so either $w_{j} \equiv v_{j}$ or $w_{j}-v_{j}$ takes on both positive and negative values. Suppose $w_{j_{1}}>v_{j_{1}}, w_{j_{2}}<v_{j_{2}}$.

We first consider $1>\eta=p / q>0$ and let

$$
u_{j}^{n+q}=G^{q}\left(u_{j+q}^{n}, \ldots, u_{j-q}^{n}\right)=\left(T u^{n}\right)_{j-p} .
$$

Since equality holds in (4.23) for $\eta=p$, and since $G_{0}^{q}>0$, it follows that

$$
\left(T^{N} w\right)_{j_{1}-N_{p}}>v_{j_{1}-N p}, \quad\left(T^{N} w\right)_{j_{2}+N_{p}}<v_{j_{2}+N p}
$$

for any nonnegative integer $N$. By condition $\mathrm{E}$, we know there exists an integer $j_{0}$ with $f_{-}^{\prime}\left(v_{j}\right)=f_{-}^{\prime}\left(v_{j}\right)=0$ if $j \leqslant j_{0}$, and by monotonicity the same is true for $\left(T^{n} u\right)_{j}$, and hence $w_{j}$. Let $N_{0}$ be chosen large enough so that $j_{1}-N_{0} p<j_{0}$. We claim that $\left(T^{N} w\right)_{j}>v_{j}$ if $N \geqslant N_{0}, j \leqslant j_{0}$. This is a contradiction, hence $w_{j} \equiv v_{j}$.

We prove the claim as follows. Suppose $\exists N_{1} \geqslant N_{0}$ and $j_{3}<j_{0}$ with $\left(T^{N{ } w}\right)_{j_{3}}<$ $v_{j_{3}}$. We also have $\left(T^{\left.N_{1} w\right)_{j_{1}-N_{p}}}>v_{j_{1}-N_{p}}\right.$.

Now we let $\bar{j}=\max \left(j_{3}, j_{1}-N p\right)-p$ and choose $R$ so large that $R q-p>$ $\left|j_{3}-j_{1}+N p\right|$. We then form

$$
T^{R}\left(T^{N_{1}}\right)_{j}-T^{R} v_{j}=\sum_{\nu=-R q}^{R q} G_{v}^{R q}\left(T^{N_{1}} w_{\nu+p+j}-v_{\nu+p+j}\right),
$$

where each partial derivative $G_{\nu}^{R q}$ is strictly positive for $-R q<\nu<0$, hence

$$
\left|T^{R}\left(T^{N_{1}} w\right)_{j}-T^{R} v_{j}\right|<\sum_{\nu=-R q}^{R q} G_{\nu}^{R q}\left|T^{N_{1}} w_{\nu+p+j}-v_{\nu+p+j}\right|
$$

which is a contradiction.

The proof for $-1<\eta<0$ follows analogously. 
For $\eta=0$ and $f \in \mathcal{C}$, we use the results of part (c) of Theorem (4.3). Define $w_{j}$ in the usual fashion. We claim first that if $w_{j}>\bar{u}$ for $j<j_{0}$, then $w_{j} \equiv u^{\boldsymbol{L}}$ for $j=j_{0}$. Suppose instead that $\bar{u}<w_{j_{1}}<u^{L}$ for $j_{1}<j_{0}-1$. Then let $\left\{v_{j}^{(1)}\right\}$ be a discrete shock for which $v_{j}^{(1)} \equiv u^{L}$ for $j \leqslant j_{1}-1, \bar{u}<w_{j_{1}}<v_{j_{1}}^{(1)}<u^{L}$, and $v_{j_{1}+1}^{(1)}<$ $\bar{u} \leqslant w_{j_{1}+1}$. It is easy to obtain the contradiction

$$
\left\|T w-v^{(1)}\right\|_{1}<\left\|w-v^{(1)}\right\|_{1}
$$

by letting $j=j_{1}+1, \mu=1$, and $\eta=0$ in (4.23).

We now call $j_{0}$ the largest integer for which $w_{j}>\bar{u}$. Thus, $w_{j_{0}+1}<\bar{u}<w_{j_{0}}$ and $w_{j} \equiv u^{L}$ for $j<j_{0}$. Let $\left\{v_{j}\right\}$ be the unique discrete shock agreeing with $\left\{w_{j}\right\}$ for $j<j_{0}$ such that $v_{j_{0}+1}<\bar{u}$. We claim $v_{j_{0}+1}=w_{j_{0}+1}$. If for example $\bar{u}>v_{j_{0}+1}>$ $w_{j_{0}+1}$, we can find another discrete shock $\left\{v_{j}^{(2)}\right\}$ for which $\bar{u}>v_{j_{0}+1}^{(2)}>w_{j_{0}+1}$ and $\bar{u}<v_{j_{0}}^{(2)}<w_{j_{0}}$ again leading to the same contradiction as above.

We need only show $w_{j} \equiv u^{R}$ for $j>j_{0}+2$. Let $j_{2}$ be the smallest integer $>j_{0}+2$ for which $w_{j_{2}}>u^{R}$. Let $\left\{v_{j}^{(3)}\right\}$ be another discrete shock for which $w_{j}<v_{j}^{(3)}$ for $j \leqslant j_{2}-2, w_{j_{2}-1}<v_{j_{2}-1}^{(3)}, v_{j_{2}}^{(3)}<\min \left(w_{j_{2}}, \bar{u}\right)$. By letting $v_{j_{2}-1}^{(3)} \searrow w_{j_{2}-1}$, we easily obtain

$$
\begin{aligned}
(T w)_{j_{2}-1}-v_{j_{2}-1}{ }^{(3)}= & w_{j_{2}-1}-v_{j_{2}-1}{ }^{(3)}-\lambda \int_{w_{j_{2}-2}}^{w_{j_{2}-1}} \chi(s) f^{\prime}(s) d s \\
& -\lambda \int_{w_{j_{2}-1}}^{w_{j_{2}}}(1-\chi(s)) f^{\prime}(s) d s \\
& -\lambda \int_{w_{j_{2}}}^{\left.v_{2}^{3}\right)}(1-\chi(s)) f^{\prime}(s) d s>0,
\end{aligned}
$$

while $\dot{w}_{j_{2}-1}<v_{j_{2}-1}^{(3)}$, which again yields a contradiction. Thus, $v_{j} \equiv w_{j}$ and we are finished.

Proof (Theorem (4.3)). For $s>0$, we have

$$
\begin{aligned}
u^{R} & \leqslant u_{j}=G\left(u_{j+1+s \lambda}, u_{j+s \lambda} u_{j-1+s \lambda}\right)=G^{q}\left(u_{j+q+q s \lambda}, \ldots, u_{j-q+q s \lambda}\right) \\
& =G^{q}\left(u_{j+q+q s \lambda}, \ldots, u_{j+q s \lambda}\right)<G^{q}\left(u_{j+q s \lambda}, \ldots, u_{j+q s \lambda}\right)=u_{j+q s \lambda}
\end{aligned}
$$

if $u_{j}$ is such that $f^{\prime}\left(u_{\nu}\right) \leqslant 0$ for $\nu \geqslant j+s \lambda-1$. We used monotonicity in the second inequality above.

Let $q \rightarrow \infty$. It follows that $u_{j}=u^{R}$.

The result for $s<0$ follows in the same fashion.

For $s=0$, we wish to solve

$$
\Delta_{-} f_{+}\left(u_{j}\right)+\Delta_{+} f_{-}\left(u_{j}\right)=0 .
$$

Sum this from $-\infty$ to $j$, arriving at

$$
f_{+}\left(u_{j}\right)+f_{-}\left(u_{j+1}\right)=f_{-}\left(u^{L}\right)+f_{+}\left(u^{L}\right)=f\left(u^{L}\right),
$$

where for convenience we took $\bar{u}=0=f(0)$.

Thus, if $u_{j}<0$ for $j<j_{0}$ with $u_{j_{0}+1}<0$, we must have $u_{j} \equiv u^{L}$ for $j<j_{0}-1$, while $f_{+}\left(u_{j_{0}}\right)+f_{-}\left(u_{j_{0}+1}\right)=f\left(u^{L}\right)$. Moreover, $f_{-}\left(u_{j_{0}+2}\right)=f\left(u^{L}\right)=f\left(u^{R}\right)$, so $u_{j_{0}+2}=$ $u^{R}$, and hence $u_{j} \equiv u^{R}$ for $j>j_{0}+2$.

V. Numerical Examples. In this section we shall present results from numerical computations with the algorithms discussed in the previous sections. We shall focus 
on approximations of shock solutions to the model equation

$$
u_{t}+\left(\frac{1}{2} u^{2}\right)_{x}=0
$$

The schemes (1.3) and (1.6) have also been applied to a number of other problems including the small disturbance equation of transonic flow (1.2) in two space dimensions. Results from computations with the first order scheme (1.3) as one step in a dimensional splitting algorithm were given in [4]. In a forthcoming paper we shall present transonic flow calculations with the second order scheme (1.6) and also analyze nonlinear stability of the time-discretized higher order scheme (5.5).

We have computed approximations to solutions of problems with other convex functions $f$ than that given in (5.1). The qualitative behavior in each of these approximations was essentially the same as those with the quadratic flux function.

For the first order scheme (1.3), we use an Euler approximation in time [4]. The time derivatives in the second order algorithms are approximated by Lax-Wendroff type differencing. With this discretization, the schemes will be of second order also in the time direction. The appropriate linear stability analysis is given in Section 2, Theorem 2.2.

We shall compare solutions of the one-sided schemes with solutions of the standard centered Lax-Friedrichs and Lax-Wendroff schemes. Below are the explicit formulas for the algorithms approximating a nonlinear conservation law (1.1),

$$
\begin{gathered}
u_{j}^{n+1}=\frac{1}{2}\left(u_{j+1}^{n}+u_{j-1}^{n}\right)-\lambda \Delta_{0} f\left(u_{j}^{n}\right) \quad \text { (Lax-Friedrichs) } \\
u_{j}^{n+1}=u_{j}^{n}-\lambda \Delta_{0} f\left(u_{j}^{n}\right) \\
+\frac{\lambda^{2}}{2} \Delta_{+}\left(f^{\prime}\left(\frac{1}{2}\left(u_{j}^{n}+u_{j-1}^{n}\right)\right) \Delta_{-} f\left(u_{j}^{n}\right)\right) \quad \text { (Lax-Wendroff) } \\
u_{j}^{n+1}=u_{j}^{n}-\lambda\left(\Delta_{+} f_{-}\left(u_{j}^{n}\right)+\Delta_{-} f_{+}\left(u_{j}^{n}\right)\right) \quad(1 \mathrm{st} \text { order, one-sided, (1.3)) } \\
u_{j}^{n+1}=u_{j}^{n}-\lambda\left(\Delta_{+} f_{-}\left(u_{j}^{n}\right)-\frac{1}{2} \Delta_{+}\left(f_{-}^{\prime}\left(z_{j}^{n}\right) \Delta_{+} u_{j}^{n}\right)\right. \\
\left.+\Delta_{-} f_{+}\left(u_{j}^{n}\right)+\frac{1}{2} \Delta_{-}\left(f_{+}^{\prime}\left(w_{j}^{n}\right) \Delta_{-} u_{j}^{n}\right)\right)
\end{gathered}
$$

(2nd order, one-sided, approximation of (1.6)),

$$
\begin{array}{r}
+\frac{\lambda^{2}}{2}\left(\Delta_{+}\left(f_{-}^{\prime}\left(z_{j}^{n}\right) \Delta_{+} f_{-}\left(u_{j}^{n}\right)\right)+\Delta_{-}\left(f_{+}^{\prime}\left(w_{j}^{n}\right) \Delta_{-} f_{+}\left(u_{j}^{n}\right)\right)\right) \\
\text { (2nd order, one-sided, approximation } \\
u_{j}^{n+1}=u_{j}^{n}-\lambda\left(\left(\Delta_{+}-\frac{1}{2} \Delta_{+}^{2}\right) f_{-}\left(u_{j}^{n}\right)+\left(\Delta_{-}+\frac{1}{2} \Delta_{-}^{2}\right) f_{+}\left(u_{j}^{n}\right)\right) \\
+\frac{\lambda^{2}}{2}\left(\Delta_{+}\left(f_{-}^{\prime}\left(u_{j}^{n}\right) \Delta_{+} f_{-}\left(u_{j}^{n}\right)\right)+\Delta_{-}\left(f_{+}^{\prime}\left(u_{j}^{n}\right) \Delta_{-} f_{+}\left(u_{j}^{n}\right)\right)\right) \\
\quad(2 \mathrm{nd} \text { order, one-sided, approximation } \\
u_{j}^{n+1}=u_{j}^{n}-\lambda\left(\Delta_{+} f_{-}\left(u_{j}^{n}\right)-\frac{1}{2} \Delta_{+}\left(f_{-}^{\prime}\left(\zeta_{j}^{n}\right) \Delta_{+} u_{j}^{n}\right)\right. \\
\left.+\Delta_{-} f_{+}\left(u_{j}^{n}\right)+\frac{1}{2} \Delta_{-}\left(f_{+}^{\prime}\left(\omega_{j}^{n}\right) \Delta_{-} u_{j}^{n}\right)\right) \\
+\frac{\lambda^{2}}{2}\left(\Delta_{+}\left(f_{-}^{\prime}\left(\zeta_{j}^{n}\right) \Delta_{+} f_{-}\left(u_{j}^{n}\right)\right)+\Delta_{-}\left(f_{+}^{\prime}\left(\omega_{j}^{n}\right) \Delta_{-} f_{+}\left(u_{j}^{n}\right)\right)\right)
\end{array}
$$

(2nd order, one-sided, approximation of (1.5)),

(2nd order, upwind biased, approximation of (3.8)). 
The notation which is used in (5.2)-(5.7) is defined earlier in this paper. Let us here recall a few of the more important definitions.

$$
\lambda=\Delta t / \Delta x ; \quad u_{j}^{n} \sim u\left(x_{j}, t^{n}\right), \quad x_{j}=j \Delta x, \quad t^{n}=n \Delta t .
$$

$\Delta_{0}=\frac{1}{2}\left(\Delta_{+}+\Delta_{-}\right), \Delta_{+}$and $\Delta_{-}$are the standard backward and forward difference operations, respectively. For convex $f$ with $f^{\prime}(\vec{u})=0$, we have

$$
f_{+}(u)=f(\max (u, \bar{u})), \quad f_{-}(u)=f(\min (u, \bar{u})) .
$$

See (1.3) for the definition of $f_{+}$and $f_{-}$for nonconvex $f$. See (3.5) for the definition of $z_{j}, w_{j}$ and (3.8) for the definition of $\zeta_{j}, \omega_{j}$.

In Figure 5.1 we present results with all the schemes (5.2)-(5.7) approximating a nonsteady shock with $f=\frac{1}{2} u^{2}, u(-\infty)=u_{L}=2$, and $u(\infty)=u_{R}=-1$. We have chosen $\lambda$ small $(=0.1)$ in order to mimic the time-continuous problem which is analyzed in the earlier sections. The discrete solutions after 90 time iterations are plotted.
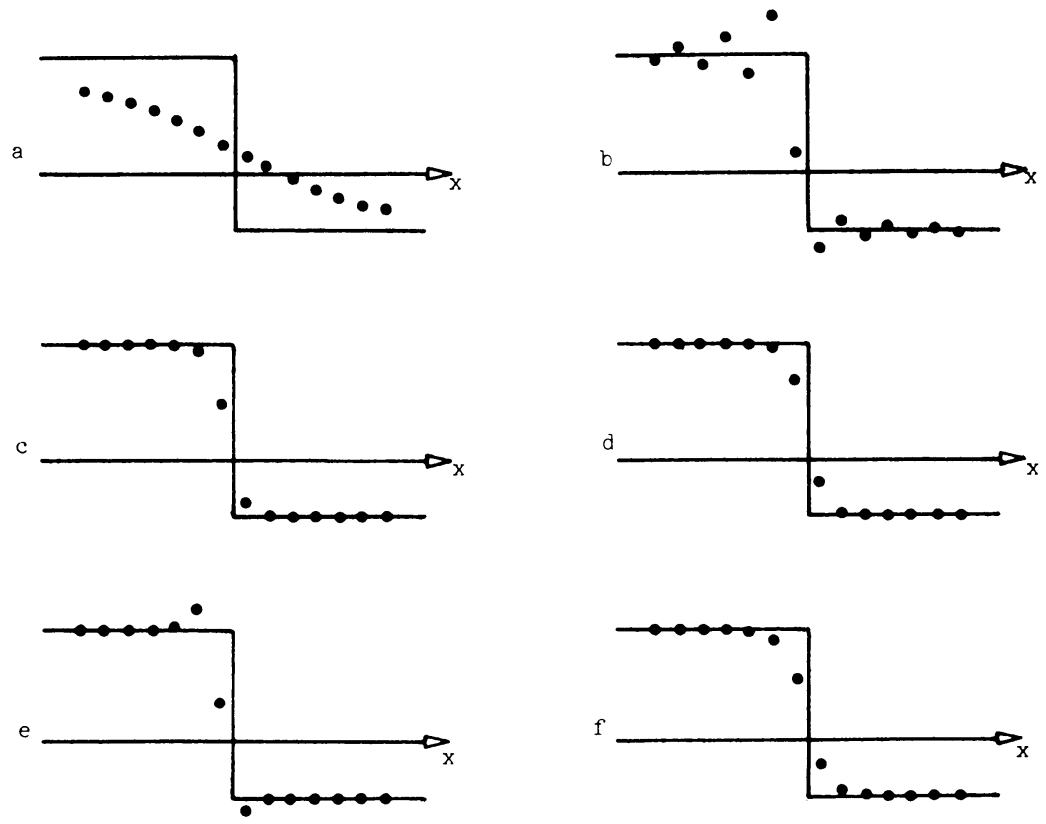

Figure 5.1

The computed solution $u$ as $a \lambda=0.1$, discrete function of $x$. Methods (5.2)(5.7). $u_{L}=2, u_{R}=0.5, f=1 / 2 u^{2}$. The solid line is the analytic solution.

The result from this test is typical for shock calculations where $f^{\prime}$ switches sign from one side to the other over the shock. The Lax-Friedrichs scheme produces a monotone profile. Since $\lambda$ is small, the dissipation is large. Calculations with larger $\lambda$ give sharper profiles. The overshoots $\left(u_{j}>2, u_{j}<-1\right)$ in the Lax-Wendroff approximation are typical for second order centered methods.

Both the first and the second order one-sided schemes (5.4), (5.5) have solutions with sharp and monotone profiles. Note in particular

(1) The shock profile is sharp on both sides. Theorem 4.3 guarantees $u_{j} \equiv u_{R}$ for increasing $j$ away from the shock. The example shows that also $u_{j} \rightarrow u_{L}$ rapidly as the index $j$ decreases. 
(2) The solution to the second order scheme is monotone as a function of $j$. For steady solutions, this is proved in Theorem 3.1.

The simplest second order one-sided method (5.6) produces a solution with overshoots. The last frame in Figure 5.1 displays the solution of (5.7). This solution is similar to that of (5.5).

We shall complement Figure 5.1 with displays of a few other cases for some of the schemes with different values of $\lambda, u_{L}$ and $u_{R}$. Figure 5.2 contains the steady problem $u_{L}=1, u_{R}=-1$ for the methods (5.3), (5.4), (5.5) and (5.6) with varying $\lambda$. The computational stability limit for the Lax-Wendroff scheme and the first order one-sided scheme was $\lambda \leqslant 1$. In these experiments the second order one-sided scheme (5.5) could be used for all $\lambda \leqslant 2$, and the scheme (5.6) was only stable for $\lambda \leqslant 0.4$.
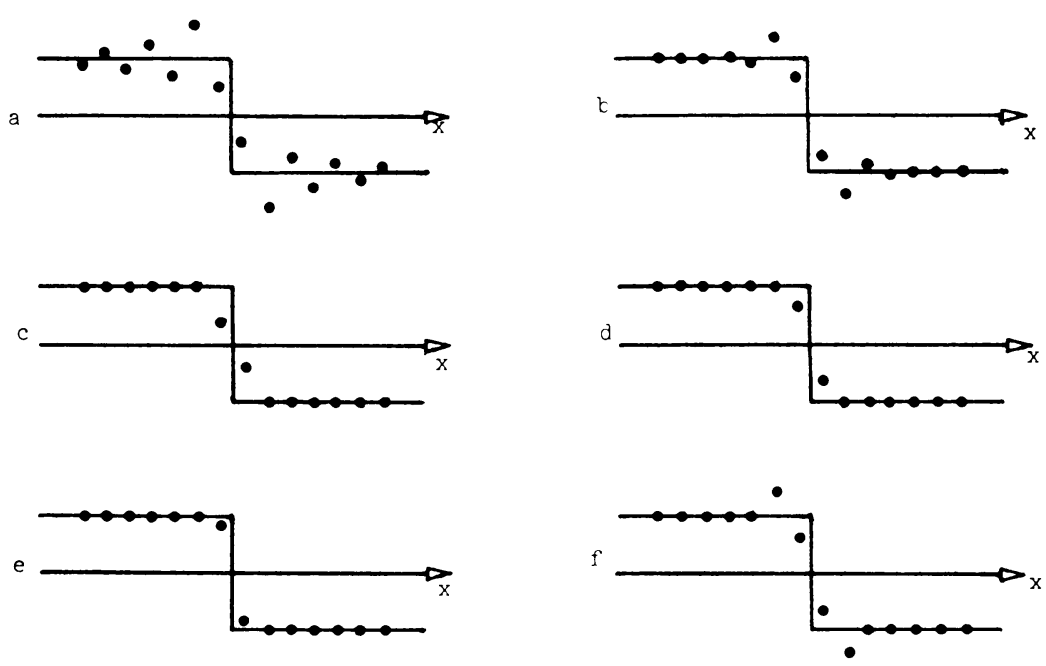

FiguRE 5.2

The computed solution $u$ as a discrete function of $x . \mathrm{a}, \mathrm{b}:$ Method (5.3) $\lambda=$ 0.1, 0.9. c, d, e: $\operatorname{Method}(5.5) \lambda=0.1,0.9,1.5$. f: $\operatorname{Method}(5.6) \lambda=0.1 . u_{L}=$ $1, u_{R}=-1, f=1 / 2 u^{2}$. The solid line is the analytic solution.

The one-sided schemes were designed to approximate solutions to nonlinear conservation laws where $f^{\prime}$ changes sign across a shock. If this is not the case, some of the desirable properties of the schemes will fail.
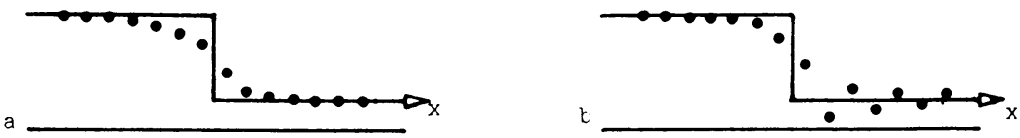

FiguRE 5.3

The computed solution $u$ as a discrete function of $x$. a: Method (5.4). b: $\operatorname{Method}(5.5) \lambda=0.1 \cdot u_{L}=2, u_{R}=0.5, f=1 / 2 u^{2}$. The solid line is the analytic solution. 
In Figure $5.3 u_{L}=2$ and $u_{R}=0.5$ with $f^{\prime}>0$ for all values of $x$.

All the traveling wave solutions that we computed were not sensitive to the choice of initial data.

The techniques and results discussed in this section have direct application for some transonic flow calculations; compare [4]. They may also be considered as models for more complex applications.

Department of Mathematics

University of California

Los Angeles, California 90024

1. M. G. Crandall \& A. Majda, "Monotone difference approximations for scalar conservation laws," Math. Comp., v. 34, 1980, pp. 1-21.

2. G. DAhLQUIST, "A special stability problem for linear multistep methods," BIT, v. 3, 1963, pp. 27-43.

3. G. DAhLQUST, "Positive functions and some applications to stability questions for numerical methods," Recent Advances in Numerical Analysis (C. de Boor and G. Golub, Eds.), Academic Press, New York, 1978, pp. 1-29.

4. B. ENGQUIST \& S. OSHER, "Stable and entropy satisfying approximations for transonic flow calculations," Math. Comp., v. 34, 1980, pp. 45-75.

5. A. HARTEN, "The artificial compression method for computation of shocks and contact discontinuities," Comm. Pure Appl. Math., v. 30, 1977, pp. 611-638.

6. A. Harten, J. M. Hyman \& P. D. LaX, "On finite difference approximations and entropy conditions for shocks," Comm. Pure Appl. Math., v. 29, 1976, pp. 297-332.

7. A. JAMrSon, "A numerical solution of nonlinear partial differential equations of mixed type," Numerical Solutions of Partial Differential Equations III, Academic Press, New York, 1976, pp. 275-320.

8. G. Jennings, "Discrete shocks," Comm. Pure Appl. Math., v. 27, 1974, pp. 25-37.

9. J. A. KRUPP \& J. D. Cole, Studies in Transonic Flow IV, Unsteady Transonic Flow, UCLA Eng. Dept. Rep., 76/04, 1976.

10. P. D. LAX, "Shock waves and entropy," Contributions to Nonlinear Functional Analysis (E. H. Zarantonello, Ed.), Academic Press, New York, 1971, pp. 603-634.

11. P. D. Lax \& B. Wendroff, "Systems of conservation laws," Comm. Pure Appl. Math., v. 23, 1960, pp. 217-237.

12. E. M. Murman \& J. D. Cole, "Calculations of steady transonic flows," AIAA J., v. 9, 1971, pp. $114-121$.

13. P. Ronche, Computational Fluid Dynamics, Hermosa, Albuquerque, N.M., 1972.

14. J. Steger, "Coefficient matrices for implicit finite difference solutions of the inviscid fluid conservation law equations," Comput. Methods Appl. Mech. Engrg., v. 13, 1978, pp. 175-188.

15. G. Strang, "Accurate partial difference methods. II: Non-linear problems," Numer. Math., v. 6, 1964, pp. 37-46.

16. B. VAN LeER, "Towards the ultimate conservative difference scheme III-Upstream-centered finite-difference schemes for ideal compressible flow," J. Comput. Phys., v. 3, 1977, pp. 263-275.

17. B. VAN LeER, "Towards the ultimate conservative difference scheme IV; A new approach to numerical convection," J. Comput. Phys., v. 23, 1977, pp. 276-299.

18. R. F. WArming \& R. M. BeAM, "Upwind second-order difference schemes and applications in aerodynamical flows," AIAA J., v. 14, 1976, pp. 1241-1249.

19. R. F. WARMING \& R. M. BEAM, "On the construction and application of implicit factored schemes for conservation laws," Computational Fluid Dynamics, SIAM-AMS Proceedings, vol. 11, 1978, pp. $85-129$. 